TITLE:

\title{
Attachment of solid elongated particles on the surface of a stationary gas bubble
}

\section{AUTHOR(S):}

Lecrivain, Gregory; Petrucci, Giacomo; Rudolph, Martin; Hampel, Uwe; Yamamoto, Ryoichi

\section{CITATION:}

Lecrivain, Gregory ... [et al]. Attachment of solid elongated particles on the surface of a stationary gas bubble. International Journal of Multiphase Flow 2015, 71: 83-93

\section{ISSUE DATE:}

2015-05

URL:

http://hdl.handle.net/2433/196088

\section{RIGHT:}

2015 Elsevier Ltd. NOTICE: this is the author's version of a work that was accepted for publication in International Journal of Multiphase Flow. Changes resulting from the publishing process, such as peer review, editing, corrections, structural formatting, and other quality control mechanisms may not be reflected in this document. Changes may have been made to this work since it was submitted for publication. A definitive version was subsequently published in International Journal of Multiphase Flow, 71, 2015, doi:10.1016/j.jimultiphaseflow.2015.01.002; この論文は出版社版であ りません。引用の際には出版社版をご確認ご利用ください。; This is not the published version. Please cite only the published version. 
Highlights:

- Attachment of elongated particles on a gas bubble is investigated

- Translational particle velocities are compared with simulated data

- Two kinds of attachments, which depend on the collision area, are discovered

- Results are relevant to three-phase flow separation processes such as flotation 


\section{Attachment of solid elongated particles on the surface of a stationary gas bubble}

Gregory Lecrivain ${ }^{1,2, \dagger}$, Giacomo Petrucci ${ }^{2,3}$, Martin Rudolph $^{4}$, Uwe Hampel ${ }^{2,5}$ and Ryoichi Yamamoto ${ }^{1}$

$$
\begin{gathered}
{ }^{1} \text { Kyoto University, } \\
\text { Department of Chemical Engineering, } \\
\text { Kyoto 615-8510, } \\
\text { Japan. } \\
{ }^{2} \text { Helmholtz-Zentrum Dresden-Rossendorf, } \\
\text { Institute of Fluid Dynamics, } \\
\text { Bautzner Landstraße 400, } \\
\text { 01328 Dresden, } \\
\text { Germany. } \\
\text { Italy. } \\
\text { Dipartimento di Ingegneria "Enzo Ferrari", } \\
\text { Via Vignolese, 905/B, } \\
41125 \text { Modena, }
\end{gathered}
$$
Helmholtz-Institute Freiberg for Resource Technology,

Halsbrücker Str. 34, 09599 Freiberg, Germany.
${ }^{5}$ Technische Universität Dresden Institut für Energietechnik

AREVA-Stiftungsprofessur für Bildgebende Messverfahren für die Energie- und Verfahrenstechnik, 01062 Dresden,

Germany.

†corresponding author:

Tel. : +81-(0)75-383-2651

Fax. : +81(0)75-383-2651

g.lecrivain@hzdr.de 


\section{Abstract:}

2 Froth flotation is a separation process which plays a major role in the mining industry. It is 3 essentially employed to recover a vast array of different valuable commodities such as rare earth

4 minerals essential to the manufacture of high-tech products. Owing to its simplicity, the process

5 is also widely used for de-inking recycled paper fibres and for waste water treatment. The

6 flotation process essentially relies on the attachment of solid particles on the surface gas bubbles

7 immersed in water. The present study seeks to investigate the effect of the particle shape on the

8 attachment mechanism. Using an in-house optical micro-bubble sensor the approach, the sliding

9 and the adhesion of micron milled glass fibres on the surface of a stationary air bubble immersed

10 in stagnant water is thoroughly investigated. The translational and rotational velocities were

11 measured for fibres of various aspect ratios. The results are compared with a theoretical model

12 and with experimental data obtained with spherical glass beads. It is found that the fibre

13 orientation during the sliding motion largely depends on the collision area. Upon collision near

14 the upstream pole of the gas bubble the major axis of the fibre aligns with the local bubble

15 surface (tangential fibre alignment). If collision occurs at least $30^{\circ}$ further downstream only head

16 of the fibre is in contact with the gas-liquid interface (radial fibre alignment).

18 Keywords: Froth flotation, three-phase system, solid elongated particles, particle attachment, gas-liquid interface. 


\section{Introduction}

\subsection{Motivation}

23 Froth flotation is a versatile separation process which plays a major role in the mining industry. It

24 is essentially employed to recover a vast array of different valuable commodities such as copper, zinc, nickel, phosphate and rare earth minerals essential to the manufacture of high-tech products (Fuerstenau, Jameson et al., 2007). Owing to its simplicity, the process has more recently seen widespread applications in the non-mining field. Flotation is for instance used for the de-inking of recycled paper fibres (Kemper, 1999) and for the removal of pollutants from waste water (Rubio, Souza et al., 2002). In mineral froth flotation the separation can be accomplished in a flotation cell, which is essentially a tank fitted with an impeller (Ahmed and Jameson, 1985). The impeller disperses air into fine gas bubbles and agitates the slurry. It provides a favourable environment in the cell for the promotion of bubble collision with the finely ground ore

33 (Fuerstenau, Jameson et al., 2007). Typical values of particle diameters, for which the recovery 34 rate is high, vary from approximately $d_{p}=10 \mu \mathrm{m}$ to $d_{p}=150 \mu \mathrm{m}$ (Tao, 2005, Jameson, 2010).

35 Many ore minerals are naturally hydrophilic. The addition of so-called "collectors" to the slurry, which are absorbed by the mineral surface, renders the precious mineral particles hydrophobic (Rosenqvist, 2004). The hydrophobised particles then attach to the surface of the rising bubbles, whose size generally ranges from $d_{b}=0.6$ to $2 \mathrm{~mm}$ in diameter (Rubio, Souza et al., 2002). The particle-bubble aggregates are conveyed to the top of the flotation cell to form a rich mineralladen froth layer, which eventually overflows into a launder as a separate product. Since pure liquids generally do not foam, "frothers" are utilised to control the bubble size and to stabilise the froth (Cho and Laskowski, 2002). The gangue, i.e. the commercially valueless hydrophilic material, eventually exits the flotation cell as slurry.

\subsection{Particle shape}

46 Ore grinding in froth flotation is an important step to liberate the valuable mineral particles from

47 the gangue (Forssberg, Subrahmanyam et al., 1993). Kursun and Ulusoy (2006) showed for

48 instance that the shape of talc mineral particles produced by milling considerably deviated from

49 an ideal sphere. Talc minerals ground by rod milling showed higher elongation and flatness than

50 those ground by ball milling. The study of Rahimi, Dehghani et al. (2012) also suggested that rod

51 milling caused an elongation of the particles and that ball milling caused a greater particle

52 roundness. Various studies have shown that the particle elongation increases the recovery rate. 
53 Koh, Hao et al. (2009) found that ground ballotini particles had a higher recovery rate than

54 spherical ballotini particles. The work of Yekeler, Ulusoy et al. (2004) also corroborated this

55 finding. The team experimentally observed that particle elongation increased the ease, with which

56 a particle attaches to a bubbles surface. Particle roundness tended to have an adverse effect on the

57 recovery rate. Note, that the recovery rate $k$ is the rate at which the desired particles are

58 recovered from the suspension. In a flotation tank, the number concentration $c(t)$ of the desired

59 particles will decay exponentially with time. Ahmed and Jameson (1985) suggested the following

60 formula $c(t)=c_{0} \exp (-k t)$, where $c_{0}$ was the initial concentration in the tank.

61

\section{1.3. Particle attachment on bubble surface}

63 The attachment of a solid particle on the surface of a gas bubble can be divided into three 64 successive stages: the particle approach, the collision with the bubble and the sliding down the gas-bubble interface (Schulze, 1989, Albijanic, Ozdemir et al., 2010). The downward sliding motion of the particle is caused by the gravity and also by the hydrodynamic forces arising from the local water flow around the rising bubble. Should the particle approach the bubble surface within the range of attractive surface forces, a thin intervening liquid film between the gas-liquid interface and the solid-liquid interface forms. The liquid film eventually drains, leading to a critical thickness at which rupture occurs (Ralston, Fornasiero et al., 1999). The rupture of the liquid film results in the formation of a stable three-phase contact (Schulze, 1992). The deployment of high speed camera systems has been favoured in recent years to observe the particle attachment in great detail. Wang, Zhou et al. (2003a) photographically recorded the attachment of free falling spherical glass beads on a stationary air bubble, which had undergone various surface treatments. Gu, Sean Sanders et al. (2004) investigated the attachment of rising spherical hydrogen bubbles on a larger fixed bitumen particle. Hubička, Basařová et al. (2013) measured the trajectories of an approaching large solid spherical particle on a stationary gas bubble. The sliding was however left out by the authors. Verrelli, Bruckard et al. (2014) were the first to look at the attachment of non-spherical particles. They measured the induction time, i.e. the time required for the liquid film to thin to its critical film thickness (Ye, Khandrika et al., 1989), of 'angular frit' particles falling on a gas bubble. The above state of the art clearly shows

82 that the attachment process has largely been limited to the attachment of perfectly spherical 83 particles. The effect of shape irregularity on the attachment process has received scarce attention.

84 To date only one attempt can be found in the literature. The present piece of work aims to 
85 alleviate this shortcoming by experimentally investigating the attachment of elongated particles

86 with an aspect ratio of up to 7.

\section{Methods}

\subsection{Experimental facility}

90 The experimental procedure employed to visualise attachment of solid particles was inspired by

91 the work of Verrelli, Bruckard et al. (2014). As illustrated in Figure 1 the experimental set-up

92 essentially consisted of water tank in which a needle was placed in a horizontal position. The 25

93 gauge ultra-smooth hydrophobic needle with a ta-C diamond-like carbon coating (SGE

94 Analytical Science, Diamond MS Syringe 0355321) was attached to a $50 \mu$ l precision syringe

95 (Hamilton, 1805RNSYR). It allowed blowing a stable and stationary air bubble, whose diameter

96 could be varied from $d_{b}=1.3 \mathrm{~mm}$ to $d_{b}=1.7 \mathrm{~mm}$. A larger bubble diameter resulted in a

97 detachment of the gas bubble from the needle. The adhesion force holding the bubble on the

98 needle tip could no longer overcome the buoyancy force. The present bubble size range matched

99 the typical size range frequently found in other literature data (Huang, Legendre et al., 2011). The

100 water was kept at a constant room temperature of $20{ }^{\circ} \mathrm{C}$ and had a $\mathrm{pH}$ value of 7.8 . The opened-

101 water tank was made of transparent Plexiglas walls. A tube fastened in a vertical position had its

102 immersed extremity placed $15 \mathrm{~mm}$ away from the bubble upper pole (henceforth referred to as

103 the upstream pole), which corresponded to a distance of about 10 bubble diameters. The reason

104 behind the use of the fastened vertical tube was threefold: 1. to guide the particles all the way

105 down to the gas bubble, 2. to avoid an interference of the falling particle with the surrounding

106 liquid and 3. to hold the Pasteur pipette in a stable vertical position. The Pasteur pipette,

107 containing the particles heavily diluted in water, could then be placed in the tube in question. The

108 bulb of the pipette in contact with ambient air was pierced to avoid a squeezing that would

109 potentially give the particles an undesired acceleration. By releasing the finger from the bulb the

110 solid particles could start their decent with an initial velocity close to zero. The present facility

111 unfortunately did not exactly reproduce all mechanisms observed in an industrial flotation cell.

112 The motion of the liquid and of the gas phases were here left out, and so were the effect of frother

113 and collector addition. To the best of the authors' knowledge the present study, even though it is

114 a fairly simple system, is the first of its kind that seeks to investigate the effect of particle

115 elongation on the overall attachment mechanism, which is of chief importance in the flotation

116 process. It is hoped that the present results will help build more complex models in the future. 


\section{2.2. Solid particles}

119 Experimental tests were performed with spherical particles for comparison purposes and fibre-

120 like elongated particles. The glass spherical particles (Wiwox, STGP002) had a particle-to-liquid

121 density ratio of $S=\rho_{S} / \rho_{L}=2.45$ and a diameter ranging from $d_{p}=50 \mu \mathrm{m}$ to $d_{p}=100 \mu \mathrm{m}$.

122 The milled glass fibres (3B-Fibreglass, MF01ER) had a particle-to-liquid density ratio of $S=2.5$ 123 and a length ranging from $100 \mu \mathrm{m}$ to $200 \mu \mathrm{m}$ in the long-axis direction (major axis). Detailed 124 images of the elongated particles obtained from the on-site Scanning Electron Microscope which 125 operates under high vacuum conditions (Zeiss EVO 50) revealed an aspect ratio, defined as the 126 ratio of fibre length to diameter, ranging from $e=1$ to $e=7$. Figure 2a illustrates high127 resolution images of spherical particles. The small structures on the surface of the elongated 128 particles (Figures 2b) are probably smaller glass particles. This highly polydispersity in the 129 particle size distribution can be seen in Figure 2c.

130 A spherical particle falling in stagnant water eventually reaches its terminal velocity

$$
u_{\infty}=g\left(\frac{S d_{p}^{2}}{18 v}\right)\left(1-\frac{1}{S}\right)=g \tau\left(1-\frac{1}{S}\right)
$$

131 where $g$ is the gravity and $v=\mu / \rho_{L}$ is the liquid kinematic viscosity, i.e. the ratio of the liquid 132 dynamic viscosity to the liquid density. The terminal velocity of a spherical particle can be 133 conveniently expressed using the particle response time $\tau$. This time corresponds to the time 134 required by a particle to respond to a change in the liquid velocity (Crowe, Schwarzkopf et al., 135 2011). The response time $\tau$ is defined as

$$
\tau=\frac{S d_{p}^{2}}{18 v}
$$

136 For an elongated particle the terminal velocity will be affected by the orientation of the major 137 axis relative to direction of motion. In the present work the direction of motion simply coincides 138 with the direction of the gravity $g$ since the liquid is at rest. The terminal velocity of an elongated

139 particle with an aspect ratio of $e=6$ will differ by up to $60 \%$ to that of a spherical particle with 140 equivalent volume (Kasper, Niida et al., 1985). Further analysis on the terminal velocity of an 141 elongated particle is dealt with in greater detail in the discussion section. 


\subsection{Particle hydrophobicity}

146 The determination of the particle hydrophobicity is not straightforward and often requires special 147 instrumentation. The sessile method, one of the simplest available methods, which involves the 148 optical measurement of the contact angle of a droplet at rest on a substrate, will often fail for 149 powder (Susana, Campaci et al., 2012). In addition the sole determination of the contact angle 150 between a solid surface and a gas-liquid interface does not allow for the exact description of the 151 surface hydrophobicity since specific and aspecific interactions need to be considered. 152 Consequently, two surfaces with the same contact angle with water can actually have a different 153 hydrophobicity (van Oss, 2003). To overcome this, inverse gas chromatography (Mohammadi-

154 Jam and Waters, 2014) was presently used to measure the specific surface free energies of the 155 two sets of powder previously described in section 2.2. The inverse gas chromatography 156 measurement device (Surface Energy Analyser, Surface Measurement Systems) was here used 157 for determination of the disperse component $\gamma^{d}$, the Lewis acid component $\gamma^{+}$and the Lewis base 158 component $\gamma^{-}$of the specific surface free energy at surface coverages of $1 \%$, of $20 \%$ and of an 159 extrapolation to 100 \% (Das, Larson et al., 2011, Gamble, Leane et al., 2012). The variation in 160 the percentage of the surface coverage allowed an assessment of the heterogeneity in the surface 161 energy. The total surface free energy was then calculated as follows

$$
\gamma^{t}=\gamma^{d}+2 \sqrt{\gamma^{+} \gamma^{-}}
$$

162 Prior to the determination of the surface free energy at different surface coverages, the specific 163 surface area of the two sets of particles per unit mass $S_{m}^{B E T}$ were determined with the $\mathrm{N}_{2}$-BET 164 method (Brunauer, Emmett et al., 1938) using special instrumentation (FlowSorb II 2300,

165 Micromeritics). The specific surface area per unit mass helped determine the amount of probe 166 gases needed for a given surface coverage. For the determination of the disperse component 167 heptane, octane, nonane and decane probe gas molecules were carried by a helium gas flowing at 168 a rate of $10 \mathrm{~cm}^{3} / \mathrm{min}$. Monopolar ethyl acetate was used for the determination of the Lewis acid 169 component and dichloromethane for the Lewis base, respectively. The column was operated at 0 $170 \%$ humidity and at a $90^{\circ} \mathrm{C}$ temperature to ensure a clean surface, which in-turn guaranteed a 171 desorption throughout the entire measurement procedure. A mass sample was chosen so that the 172 surface area equalled $0.5 \mathrm{~m}^{2}$. The samples were tapped vertically for ten minutes in a 3-mm inner 173 diameter and 30-cm length column, which was sealed with inert silanized glass wool. Prior to the 174 measurement the samples were conditioned at a $100^{\circ} \mathrm{C}$ temperature for a period of 5 hours in a 
175 helium gas also flowing at $10 \mathrm{~cm}^{3} / \mathrm{min}$. The specific surface free energy components were 176 calculated using the peak maximum of the probe molecule retention. The Schultz approach was 177 employed for the disperse component $\gamma^{d}$, the van Oss-Chaudhury-Good/Della-Volpe approach 178 for the Lewis acid/basic component (Das, Larson et al., 2011). To thermodynamically evaluate 179 whether the particle attachment to a gas bubble is actually favourable in liquid water the specific 180 free energy of interaction $\Delta G_{p w b}$ was calculated as follows (van Oss, 2003)

$$
\Delta G_{p w b}=\left(\sqrt{\gamma_{S}^{d}}-\sqrt{\gamma_{G}^{d}}\right)^{2}-\left(\sqrt{\gamma_{S}^{d}}-\sqrt{\gamma_{L}^{d}}\right)^{2}-\left(\sqrt{\gamma_{G}^{d}}-\sqrt{\gamma_{L}^{d}}\right)^{2}+2\left(\sqrt{\gamma_{L}^{+}}\left[\sqrt{\gamma_{S}^{-}}+\sqrt{\gamma_{G}^{-}}-\sqrt{\gamma_{L}^{-}}\right]+\sqrt{\gamma_{L}^{-}}\left[\sqrt{\gamma_{S}^{+}}+\sqrt{\gamma_{G}^{+}}-\sqrt{\gamma_{L}^{+}}\right]-\sqrt{\gamma_{S}^{+} \gamma_{G}^{-}}-\sqrt{\gamma_{p}^{-} \gamma_{G}^{+}}\right)
$$

181 The subscript L, G and S respectively denote the specific free surface energies of the liquid water 182 (L), of the gas bubble $(\mathrm{G})$ and of the solid particles (S). Finally the contact angle $\theta$ between the 183 particle surface and the liquid water was determined using the surface free energy components as 184 follows (van Oss, 2003)

$$
\theta=\cos ^{-1}\left(\frac{2}{\gamma_{L}^{t}} \cdot\left[\sqrt{\gamma_{S}^{d} \gamma_{L}^{d}}+\sqrt{\gamma_{S}^{+} \gamma_{L}^{-}}+\sqrt{\gamma_{S}^{-} \gamma_{L}^{+}}\right]-1\right)
$$

185 The results are summarised in Table 1. The three components of the total specific energy for the 186 air bubble and for the liquid water were taken from the literature (van Oss, Giese et al., 2005, 187 VDI-GVC, 2013). Findings from the present inverse gas chromatography measurements show 188 that, for the two sets of particles and under all surface coverages, the specific free energy $\Delta G_{p w b}$ 189 is always negative and thus attachment is favourable in all cases. Thermodynamically speaking, 190 the particles are indeed hydrophobic. Irrespective of the surface coverage, the specific free energy $191 \Delta G_{p w b}$ shows little heterogeneity for the spherical particles and the values of the free specific 192 interactions and of the contact angles are all similar. The same cannot be said for the elongated 193 particles. At a surface coverage of the $1 \%$ there is a higher polarity for the elongated particles, 194 which resulted in smaller contact angles and a lower free energy of interactions with a bubble in 195 water. Compared to the spherical particles the hydrophobicity at $10 \%$ and at $100 \%$ coverage is 196 greater. It is expected from the present results that the elongated particles will attach to the gas 197 bubble slightly differently. The study also revealed a contact angle lying in the range $60^{\circ}<\theta<$ $19870^{\circ}$. Fuerstenau, Jameson et al. (2007) showed that this contact angle is large enough to achieve a 199 good floatability in the flotation process. The present contact angle slightly overestimates the 200 values worked out by Tino, Dieter et al. (1996) and by Nowak, Robbins et al. (2013) who 201 reported contact angles ranging from $50^{\circ}$ to $60^{\circ}$. The calculation of their contact angles was 
202 performed using the sessile method, which involved a water droplet was at rest on a glass

203 substrate. Last but not least, even with a surface treatment involving for instance 204 chlorotrimethylsilane, the contact angle of smooth spherical glass beads can only be increased to 205 about $90^{\circ}$ (Nowak, Robbins et al., 2013), therefore it is very hard to render glass particles fully 206 hydrophobic.

\subsection{Measurement instrumentation}

To observe the attachment of a solid particle on a gas bubble surface the in-house optical microbubble sensor (Ren, Wu et al., 2011) developed at the Helmholtz Zentrum Dresden-Rossendorf was put to use in the present study. The sensor consists of two tubular waterproof housings facing each other's extremities. The gas bubble was placed between the two tubular shafts partly

213 immersed in water. A distance of about $40 \mathrm{~mm}$, which corresponded to about 25 bubble 214 diameters, separated the two shafts. The first housing enclosed the CCD camera and the second 215 enclosed the LED illumination system. The light source flashed in synchronisation with the 216 camera exposure. A focus length of $20 \mathrm{~mm}$ allowed for a field of view of about $3.5 \times 2.6 \mathrm{~mm}^{2}$, 217 which was large enough to hold the gas bubble and capture the entire particle attachment. Each

218 image had a resolution of $640 \times 480$ pixels. With a particle terminal velocity ranging from $219 u_{\infty}=1.5 \mathrm{~mm} / \mathrm{s}$ to $u_{\infty}=7 \mathrm{~mm} / \mathrm{s}$ (See annex 1) and a frame rate of 120 images per second it 220 resulted in a local particle displacement between two consecutive illumination pulses ranging 221 from $10 \mu \mathrm{m}$ to $60 \mu \mathrm{m}$.

\subsection{Experimental data processing}

224 The footage of a particle attachment was a very tedious task. The particle had to evolve over the 225 course of the entire attachment process in the focus plane of the micro-bubble sensor. The attachment of a fibre was an even unlikelier event since the major axis also had to remain in the plane of focus so that the particle orientation could be determined. The chance of capturing the attachment of a very single particle was actually low. The facility did not allow the release of one particle at a time. Therefore only the head of the falling cloud, which had a local dilution greater than that of the bulk of the cloud, was here of interest. The reason behind the footage of the cloud head was twofold: 1 . the chance of capturing single attachment in the field of view was increased

232 and 2. the particle-induced flow and the inter-particle collisions near the gas bubble, which were 233 observed to affect the attachment, could be avoided. While it was difficult to accurately estimate 
234 the occurrence of an exploitable attachment, we found out that about 50 experimental test runs

235 resulted in one exploitable attachment. A measurement campaign lasting several weeks was 236 therefore necessary to capture the 30 attachments of elongated particles and the 4 attachments of

237 spherical particles presented in the present work. Each successful capture of a particle 238 attachment, in which the particle evolved in the focus plane, was exported in the form of an 239 image sequence to the post-processing program Fiji. The image processing program is an open240 source platform normally used for biological-image analysis (Schindelin, Arganda-Carreras et al., 241 2012). After the threshold of each image sequence, a circle was fit to the gas bubble and polar 242 coordinate system was defined. The diameter of the needle, which was of course known 243 beforehand, was used as the reference length. During the approach the orientation of the major 244 particle axis and its velocity could be automatically detected by fitting a two-dimensional 245 ellipsoid. However during the sliding the particle shape could no longer be automatically 246 discriminated from the gas bubble shadow. For this reason the determination of the major axis 247 and the velocities were done manually for various images. It resulted in a time-consuming image 248 processing.

\subsection{Spherical particle transport model}

251 The present experimental investigation was carried with "clean" bubbles, i.e. the gas-liquid 252 interface underwent no contamination by frothers, which were previously found to significantly 253 influence the collision efficiency (Sarrot, Guiraud et al., 2005). Further information on the effect 254 of partial and full interface contamination on the collision efficiency can be found in the study of 255 Legendre, Sarrot et al. (2009). The physico-chemical interfacial forces normally prevail over the 256 hydrodynamic forces only within a very short distance from the gas-liquid interface, typically for 257 a gap value lower than $h<0.1 \mu \mathrm{m}$ (Huang, Legendre et al., 2012): a distance by several order of 258 magnitude smaller than the particle size. Since the physic-chemical interfacial forces have little 259 effect on the collision they were here left out. The history force and the lift force are of second 260 order and can also be neglected (Nguyen, 2003). The effect of the Basset force even though it 261 was included in the Lagrangian particle model of Verrelli, Koh et al. (2011) is often left out in 262 other numerical studies (Nguyen and Nguyen, 2009). Thus retaining only the hydrodynamic drag 263 exerted by the liquid phase, the gravity and the buoyancy, the vectorial transport equation of a 264 point-like solid spherical particle evolving in the liquid phase therefore reads 


$$
m \frac{d \vec{u}}{d t}=-3 \pi \mu d_{p} \vec{u}+\left(m-m_{f}\right) \vec{g}
$$

265 In the above equation $\vec{u}$ is the particle velocity vector. Since the stationary bubble is immersed in 266 stagnant water the velocity of the liquid phase is set to zero throughout the simulation. The term $267 m$ corresponds to the particle mass and $m_{f}$ to the mass of liquid displaced by the particle. The 268 term $\mu$ corresponds to the dynamic viscosity of the liquid phase. The transport equation can 269 conveniently be expressed in terms of the two polar coordinates $(r, \phi)$, in which $r$ is the distance 270 from the bubble centre to the particle centre and $\phi$ the angle measured from the vertical axis (see

271 Figure 3 ). The polar axis $\phi=0$ coincides with the upstream pole of the gas bubble. Using the 272 terminal velocity $u_{\infty}$ defined in Eq. (1) the polar transformation of Eq. (6) leads to the following 273 system of scalar equations

$$
\begin{gathered}
\frac{d u_{r}}{d t}=-\left[\frac{\left(1+k_{s}\right) f_{r}}{\tau}\right] u_{r}+\left[\frac{u_{\phi}^{2}}{r}-\frac{u_{\infty}}{\tau} \cos (\phi)\right], \\
\frac{d u_{\phi}}{d t}=-\left[\frac{f_{\phi}}{\tau}+\frac{u_{r}}{r}\right] u_{\phi}+\left[\frac{u_{\infty}}{\tau} \sin (\phi)\right] .
\end{gathered}
$$

274 The radial and tangential velocities are given by

$$
u_{r}=\frac{d r}{d t} \quad, \quad u_{\phi}=r \frac{d \phi}{d t}
$$

275 The particle will deviate from its original trajectory as it approaches the bubble surface. To 276 account for the change in the particle motion towards the bubble side the hydrodynamic force 277 requires an artificial correction. Since the particle size is much smaller than the bubble size, it can 278 be assumed that the particle encounters a fairly flat gas-liquid interface (Nguyen and Jameson, 279 2005). The radial and tangential drag components are therefore corrected using the following 280 polynomial approximations (Huang, Legendre et al., 2012)

$$
\begin{gathered}
f_{r}=1+\frac{3}{2} \lambda+\frac{9}{4} \lambda^{2}+\frac{19}{8} \lambda^{3}+\frac{93}{16} \lambda^{4}+\frac{387}{32} \lambda^{5}+\frac{1197}{64} \lambda^{6}+\frac{5331}{128} \lambda^{7}+\frac{19821}{256} \lambda^{8}+\frac{76115}{512} \lambda^{9}+\frac{3}{10}\left[\frac{(2 \lambda)^{10}}{1+2 \lambda}\right], \\
f_{\phi}=1-\frac{3}{4} \lambda+\frac{9}{16} \lambda^{2}-\frac{59}{64} \lambda^{3}+\frac{465}{256} \lambda^{4}-\frac{15813}{7168} \lambda^{5}+2\left(\frac{\lambda^{6}}{1+\lambda}\right)
\end{gathered}
$$

281 where the dimensionless variable $\lambda$ equals $\lambda=0.5 d_{p} /\left(2 h+d_{p}\right)$. The gap $h=r-0.5\left(d_{p}+d_{b}\right)$ 282 corresponds to smallest distance between the particle surface and the bubble surface. Close to the 283 gas-bubble interface the particle typically experiences an increase in the hydrodynamic drag force 
284 and a decrease in the tangential force. Far from the bubble the two approximations $f_{r}$ and $f_{\phi}$ are 285 smoothly brought back to unity using a blending function to exactly achieve the correct terminal 286 velocity $u_{\infty}$. The blending function is a smooth approximation of the Heaviside step function. It 287 is here given by

$$
H_{V}\left(h^{*}\right)=\frac{1}{2}\left(1+\tanh \left[\frac{h^{*}-h_{m}^{*}}{\Delta h^{*}}\right]\right)
$$

288 The upper script ${ }^{*}$ indicates the normalisation of the gap with the reference scaling $\left(d_{b} / 2\right.$ for a 289 length variable, see Eq. 14). With the smoothing centre $h_{m}^{*}=0.6$ and the smoothing radius of $290 \Delta h^{*}=0.3$ the final correction then takes the form $f_{r, \phi}=H_{V}+\left(1-H_{V}\right) f_{r, \phi}$. The effect of the 291 blending function can be seen in Figure 4. A cut off of the polynomial function to the $3^{\text {rd }}$ order is 292 also shown. The reduction of Eq. (10-11) to a third-order polynomial will largely affect the 293 radial drag correction close to the gas-bubble interface, i.e. within $h^{*}<0.1$. As in the original 294 model of Huang, Legendre et al. (2012), the above $10^{\text {th }}$ - and $6^{\text {th }}$-order polynomial 295 approximations are here employed. Upon collision with the gas bubble the two drag correction 296 factors no longer make sense and therefore they equal unity. The dimensionless friction factor $k_{s}$ 297 is introduced to correct the particle drag force during the sliding motion on the bubble surface. 298 Wang, Zhou et al. (2003a) suggested, as is the case here, $k_{s}=0.03$ for untreated glass beads 299 interacting with with 'clean' bubbles. The value of $k_{S}$ is however significantly affected by the use 300 of collectors and frothers (Wang, Zhou et al., 2003b). Methylated glass spheres showed an 301 increase in the friction factor to $k_{S}=0.1$. A gas bubble stabilised with sodium palmitic acid 302 caused an increase in the friction factor to $k_{s}=1$. The friction coefficient therefore needs 303 particular attention since its value spans up to three orders of magnitude. During the approach the 304 friction factor $k_{s}$ is set to zero and the hydrodynamic drag force simply reduces to its well-known 305 Stokes' formulation in the far-bubble region. During the sliding, which begins when the gap $h$ 306 drops to zero, the particle is in equilibrium in the radial direction. It means that the capillary 307 adhesion force, the centrifugal force and the radial component of the gravity cancel one another 308 out and $u_{r}=0$. An extensive analysis on the adhesion forces can be found in the discussion. An 309 implicit Euler backward scheme is used for the numerical integration of the two transport 310 equations (Eq. 7-8) and a standard second-order Adams-Bashforth scheme is applied to compute 311 the particle displacement (Eq. 9). These two integration schemes were previously found 
312 appropriate to accurately compute the transport and the deposition of micron-sized particles

313 (Lecrivain and Hampel, 2012, Lecrivain, Barry et al., 2014).

\section{3. Results and Discussions}

\section{3.1. Model validation}

317 The performance of the transport model for spherical particles is initially compared with four 318 experimental reference runs, which all involved the glass beads of spherical shape. The four 319 experimental tests were carefully selected so that the corresponding collision angle $\phi_{c}$ of each 320 run lied in one of the four collision intervals $I_{i=1 . .4}$

$$
\begin{array}{cc}
I_{1}: & \phi_{c}<10^{\circ} \\
I_{2}: & 10^{\circ} \leq \phi_{c}<30^{\circ} \\
I_{3}: & 30^{\circ} \leq \phi_{c}<50^{\circ} \\
I_{4}: & \phi_{c} \geq 50^{\circ}
\end{array}
$$

321 Figure 5 presents the theoretical particle trajectories coloured by velocity magnitude with their experimental counterparts. At the initial simulation time $t=0 \mathrm{~s}$ the particle velocity equals the 323 terminal velocity calculated from Eq. (1) and the horizontal particle position $R(t=0)=R_{\infty}$

324 equals that measured from the very first occurrence of the particle in the camera field of view. 325 The distance $R$ corresponds to the shortest distance from the particle centre to the vertical rotation 326 axis of the bubble $(x=0)$. All lengths and velocity variables are henceforth made non327 dimensional with the bubble radius and with the terminal velocity so that, irrespective of the 328 changes in the bubble and particle sizes, all data can easily be compared with one another. The 329 distance $\mathrm{R}$, for instance, is normalised as follows

$$
R^{*}=\frac{2 R}{d_{b}}
$$

330 and the instantaneous particle velocity is normalised as follows

$$
u^{*}=\frac{|\vec{u}|}{u_{\infty}} .
$$

331 The terminal velocity is experimentally calculated from the two furthest upstream particle 332 positions. It can be seen that the theoretical particle trajectories and the theoretical particle 333 velocities agree qualitatively well with the experimental results. Because of the increase in the

334 drag force and the decrease in the tangential force that the particle encounters during its approach 335 near the gas-bubble interface the particle smoothly moves away from the vertical rotation axis of 
336 the bubble. The deviation of the particle trajectories can quantitatively be assessed with the

337 collision angle $\phi_{c}$. Using identical boundary conditions $\left(d_{p}, d_{b}\right)$ and initial release conditions

$338\left(u^{*}, R^{*}\right)$ it is found that the theoretical collision angles match remarkably well the experimental

339 data in the three intervals $I_{i=1 . .3}$. The model however overestimates the deviation in the particle

340 trajectory by about $15 \%$ for large collision angles. The reason behind the larger errors in the

341 fourth interval $I_{4}$ is given in the next section 3.2 .

\section{3.2. Approach of the elongated particles}

344 The velocity magnitude of each falling elongated particle during the approach phase is compared with that obtained from the four experimental runs involving the spherical particles and that obtained from the theoretical model. Figure 6 shows the velocity magnitude as a function of the polar coordinate $r^{*}$. Similarly to the model validation against the spherical glass bead data the results are sorted by collision angle and placed in one of the four collision intervals $I_{i=1 . .4}$ (Eq. 9). In each of the four subdiagrams the abscissa $r^{*}$ decreases to unity. This lower bound indicates a collision with the gas bubble surface. The results show that the velocity magnitude of the approaching particles is not significantly affected by the particle aspect ratio. It is a major finding since even a model for spherical particles can be employed to predict the translational velocities and the trajectories of elongated particles. In the far-bubble region, found to be the region in which the normalised polar radius is greater than $r^{*}>1.8$, the bubble has little effect on the particle motion: each particle descends with a constant velocity equalled to the terminal velocity $u_{\infty}$. In the bubble region $\left(r^{*}<1.8\right)$ and for a collision angle lower than $\phi_{c}<50^{\circ}$ the velocity magnitude rapidly decreases. The closer the collision near the upstream pole the greater the deceleration. Upon collision with an angle lower than $\phi_{c}<10^{\circ}$ the particle experiences a loss in velocity of about $80 \%$ (interval $I_{1}$ ). In the third interval $I_{3}$ the particle experiences a loss in velocity of about $40 \%$. As previously observed in the validation of the particle trajectories (Figure 5) the model exhibits an unrealistic behaviour for large collision angles (interval $I_{4}$ ). In

362 the fourth interval $I_{4}$ the theoretically determined velocity magnitude increases as the particle 363 approaches the bubble surface. Further analysis of the simulation data showed a strong decrease

364 in the tangential drag force component, which in-turn caused this unnatural increase in the 365 particle velocity magnitude. It seems that, for large collision angles, the two drag corrections (Eq. 366 10-11) lose validity when the particle comes very close to the gas-bubble interface, i.e. within 367 $r^{*}<1.1$. 


\subsection{Sliding of the elongated particles}

The velocity magnitude was also measured during the sliding phase. As illustrated in Figure 7, it can be seen that the particle elongation does not influence the velocity magnitude in each collision interval. The model also performs remarkably well. The velocity magnitude reaches its maximum when the polar angle reaches $100^{\circ}$. The orientation of the particle major axis with the radial direction is denoted by the angle $\gamma$ (See Figure 3 for an illustrative schematic). Figure 8 illustrates the two types of ellipsoidal attachments: the "weak attachment" and the "strong attachment". The weak attachment indicates a radial alignment of the fibre at the gas-liquid interface. Only one of the two fibre extremities eventually adheres to the gas-liquid interface ( $\gamma=0$ towards the end of the sliding). The strong attachment indicates a larger three-phase contact area. Throughout the sliding phase the particle major axis aligns with the gas-liquid interface $\left(\gamma=90^{\circ}\right)$ which results in a tangential alignment of the fibre at the gas liquid interface. The evolution of the major axis orientation as a function of the polar angle over the course of the sliding is shown in Figure 9. The figure is divided into two subfigures. The subfigure (9a) on the left hand side encompasses the elongated particles for which a weak attachment was observed. The subfigure (9b) on the right hand side encompasses the elongated particles for which a strong attachment was observed. As expected the strong attachment involves a particle orientation which equals $90^{\circ}$ throughout the entire attachment. During a weak attachment the elongated particle initially aligns with the bubble surface (tangential contact, $\gamma=90^{\circ}$ ) and when the polar angle reaches the surface bubble region $90^{\circ}<\phi<120^{\circ}$ the particle orientation suddenly changes: the contact becomes radial $\left(\gamma=0^{\circ}\right)$. Last but not least, about $90 \%$ of experimental runs, in which the collision angle exceeded the threshold $\phi_{c}>30^{\circ}$, resulted in a weak attachment (left subfigure 9a). Should the collision angle be lower than this threshold collision angle (grey area in the right subfigure $9 \mathrm{~b}$ ), the attachment was found take a strong form. The effect of the collision angle has a major effect on the fibre orientation during the sliding motion.

\subsection{Discussions}

\subsubsection{Sliding time}

397 The weak attachment is very likely due to the shorter time of "induction" (Yoon and Luttrell,

398 1989) which does not allow for the formation of a stronger tangential three-phase contact.

399 Verrelli, Koh et al. (2012) experimentally measured the induction time of methylated borosilicate 
400 glass spheres. The team showed that the induction time increased with the collision angle, i.e. a 401 larger collision angle resulted in a longer time required for the film rupture to occur. For collision 402 angles greater than $\phi_{c}>30^{\circ}$ the team estimated an induction time ranging from 0.1 to 0.2 s. A 403 collision near the upstream pole resulted in a lower induction time of about $0.02 \mathrm{~s}$. During a weak

404 attachment and irrespective of its shape, it was here found that a particle colliding with the bubble 405 at a polar angle greater than $\phi_{c}>30^{\circ}$ required on average $0.15 \mathrm{~s}$ to $0.2 \mathrm{~s}$ to reach the bubble 406 equator, after which the particle changed its orientation $\gamma$. The present sliding time needed to 407 reach the bubble equator is of course determined for glass beads with a contact angle of $\theta=$ $40860-70^{\circ}$ and can therefore not be directly compared with the induction time of glass particles 409 with a surface treatment. Yet it seems that the sliding time needed to reach the bubble equator 410 should be large enough for a strong attachment to occur.

\section{$412 \quad 3.4 .2$. Forces at the gas-liquid interface}

413 Findings from this work along with the experimental observations of Wang, Zhou et al. (2003b) 414 have shown that the maximum value, for which the rotational velocity reaches its maxima, is not 415 exactly found at $90^{\circ}$, but at a polar angle located between $90^{\circ}$ and $110^{\circ}$. Surprisingly it fairly 416 corresponds to the range, in which the particle suddenly changes its orientation $\gamma$ over the course 417 of a weak attachment (Figure 9). Could it be that the change in the particle orientation during a 418 weak attachment is triggered by the centrifugal force? It is therefore of interest to work out which 419 forces prevail during the sliding process. The magnitude of the various forces are here derived 420 from the theoretical work of Nguyen (2003). He developed a force balance model, in which a 421 spherical particle is at rest at the downstream pole of a bubble, i.e. at $\phi=180^{\circ}$. Four major static 422 forces were identified: the capillary force $F_{c a p}$, the buoyancy $F_{b}$, the pressure force $F_{p}$ and the 423 particle weight $F_{g}$. In the following each force is normalised with the surface tension $\sigma$ and the

424 capillary length $L=\sqrt{\sigma /\left(\rho_{L} g\right)}$. The mathematical formulations are also simplified as a result of 425 the small particle-to-bubble diameter ratio. The dimensionless capillary force, which tends to pull 426 the solid particle into the gas phase, equals the product of the gas-liquid surface tension with the 427 length of the three-phase contact line. Its formulation reads

$$
\frac{F_{c a p}\left(\phi=180^{\circ}\right)}{2 \pi \sigma L}=\frac{1}{2}\left(\frac{d_{p}}{L}\right) \sin \alpha \sin (\theta-\alpha)
$$


428 For the exact definition of the angle $\alpha$ the reader is referred to the original work of Nguyen 429 (2003). Typically the total adhesion force is maximum for $\alpha=28^{\circ}$. Should the centrifugal force 430 be introduced in the original force balance model of Nguyen (2003), the value of $\alpha$, for which the 431 adhesion force is maximum, will change. The force balance model of Nutt (1960) could for 432 instance be employed. It has also been shown that the for a fixed particle volume, a prolate 433 spheroid attaches even more strongly to a gas-liquid interface because of the larger particle434 interface area (Davies, Kruger et al., 2014). An exact solution of the various forces acting on the 435 particle at the gas-bubble interface is however irrelevant in the present discussion since we only 436 seek to compare their respective orders of magnitude. The hydrostatic pressure force of the liquid 437 phase above the contact area at the gas-liquid interface is given by

$$
\frac{F_{h}\left(\phi=180^{\circ}\right)}{2 \pi \sigma L}=\frac{1}{4}\left(\frac{d_{p}}{L}\right)^{2}\left(\frac{d_{b}}{2 L}-\frac{2 L}{d_{b}}\right) \sin ^{2} \alpha .
$$

438 The buoyancy force, which largely applies to the particle volume immersed in the liquid phase, is 439 defined as

$$
\frac{F_{b}\left(\phi=180^{\circ}\right)}{2 \pi \sigma L}=\frac{1}{48}\left(\frac{d_{p}}{L}\right)^{3}\left(2+3 \cos \alpha-\cos ^{3} \alpha\right) .
$$

440 The particle weight is given by

$$
\frac{F_{g}\left(\phi=180^{\circ}\right)}{2 \pi \sigma L}=\frac{1}{12}\left(\frac{d_{p}}{L}\right)^{3} S .
$$

441 Using the theoretical formulation of the centrifugal force derived by Dai, Dukhin et al. (1998), it 442 can be shown that its magnitude is proportional to $\sin ^{2} \phi$, and therefore the centrifugal force 443 reaches its theoretical maxima at the bubble equator $\phi=90^{\circ}$. Figure 7 shows that the maximal 444 sliding velocity roughly equals the terminal velocity. With a typical terminal velocity $u_{\propto}=3$ $445 \mathrm{~mm} / \mathrm{s}$ (Annex 1) and an average bubble diameter $d_{b}=1.4 \mathrm{~mm}$, a fair estimate of the centrifugal 446 force at the equator can be given by

$$
\frac{F_{c}\left(\phi=90^{\circ}\right)}{2 \pi \sigma L}=\frac{1}{48}\left(\frac{d_{p}}{L}\right)^{3}\left(\frac{2 u_{\infty}^{2}}{g d_{b}}\right) \approx 10^{-3} \frac{1}{48}\left(\frac{d_{p}}{L}\right)^{3} .
$$

447 For comparison purposes all the above forces were made function of the ratio of the particle 448 diameter to the capillary length. With a capillary length of about $L=2.7 \mathrm{~mm}$ this ratio becomes 449 very small, i.e. $d_{p} / L \ll 1$. The particle buoyancy (Eq. 18) and the particle weight (Eq. 19) are 450 therefore two orders of magnitude smaller than the capillary force. The centrifugal force (Eq. 20) 451 is by even more orders of magnitude smaller than the capillary force. It therefore seems fair to 
452 neglect the effect of the centrifugal force on the change in the fibre orientation over the course of 453 a weak attachment.

\section{3.4.3. Terminal velocity of the elongated particles}

456 In the present model it was assumed that the particles were spherical. However, the drag of an 457 elongated particle does not necessarily equal that of an equivalent sphere. The terminal velocity $458 u_{\infty}^{\|}$of an elongated particle, which has its major axis parallel to the direction of motion (particle 459 in a vertical position), will differ from the terminal velocity $u_{\infty}^{\perp}$ of the exact same particle 460 descending with its major axis normal to the relative particle motion (particle in a horizontal 461 position). Therefore the two shape factors $\kappa_{\|}$and $\kappa_{\perp}$, defined as the ratio of the terminal velocity 462 of an elongated particle to that of an equivalent sphere of same volume and density (Kasper, 463 Niida et al., 1985) are normally introduced

$$
\kappa_{\|}=\frac{u_{\infty}^{\|}}{u_{\infty}^{e q}} \quad, \quad \kappa_{\perp}=\frac{u_{\infty}^{\perp}}{u_{\infty}^{e q}} .
$$

464 In the above formulation $u_{\infty}^{e q}$ corresponds to the terminal velocity of a sphere of equivalent 465 volume. Exact, limiting and approximate solutions for the drag on spheroids at creeping flow 466 conditions using the slender-body theory were derived by Oberbeck (1876). As is the case here, a 467 creeping flow indicates that the Reynolds number based on the particle diameter and the liquid 468 viscosity is lower than unity. One of the approximations (Loth, 2008), for which the aspect ratio 469 of a needle-like ellipsoid (prolate) is greater than unity, can be found in Table 2. The shape 470 factors are calculated for an aspect ratio of 3 and 6 . The theoretical value is compared with the 471 experimental data derived from the work of Kasper, Niida et al. (1985). For a particle aspect ratio 472 of $e=3$ one can expect a maximum difference in the terminal velocity of about $30 \%$ compared 473 to that of an equivalent sphere. This difference will increase to about $60 \%$ for a particle aspect 474 ratio equalled to $e=6$. The present model, which involves spherical particles, therefore 475 underestimates the translational velocities. It is however not directly shown in the figures since 476 the velocity variables were made non-dimensional with the terminal velocity. The experimental 477 determination of the terminal velocity was taken from the first two occurrences of the particle in 478 the camera field of view. It was shown here that the particle velocity is affected within $r^{*}<1.8$.

479 Figure 8 and the two videos available in the supplementary material show that some particles 480 enter the field of view at an altitude of about $r^{*} \approx 1.5$. It is therefore likely that the terminal 481 velocity does not always exactly correspond to the one, which would be observed at a higher 
482 altitude. Unfortunately there is no better alternative for determining the exact terminal velocity of

483 the elongated particles.

\section{Conclusions}

486 The present work looked at the attachment of solid elongated particles on the surface of a stationary gas bubble immersed in stagnant water. It was shown that the particle aspect ratio has no significant effect on the translational velocities of the particles. The far-bubble region, in which the velocity of the particle is not affected by the bubble, was found to be the region in which the normalised polar radius was greater than $r^{*}>1.8$. The results matched very well those obtained numerically with spheres as long as the collision angle remained lower than $\phi_{c}<50^{\circ}$.

492 For the first time the existence of two types of attachment has been shown. Upon collision near the upstream pole of the gas bubble the major axis of the fibre aligns with the local bubble surface (tangential fibre alignment, strong attachment). If collision occurs at least $30^{\circ}$ further downstream only head of the fibre is in contact with the gas-liquid interface (radial fibre alignment, weak attachment).

\section{Acknowledgements}

500 Union Seventh Framework Program for Research and Technological Development (2007-2013)

501 under the grant agreement number 623518.

\section{References}

504 Ahmed, N. and Jameson, G. J., 1985. The effect of bubble-size on the rate of flotation of fine particles. International Journal of Mineral Processing 14, 195-215.

Albijanic, B., Ozdemir, O., Nguyen, A. V. and Bradshaw, D., 2010. A review of induction and attachment times of wetting thin films between air bubbles and particles and its relevance in the separation of particles by flotation. Advances in Colloid and Interface Science 159, 1-21.

$510 \quad$ Journal of the American Chemical Society 60, 309-319.

511 Cho, Y. S. and Laskowski, J. S., 2002. Effect of flotation frothers on bubble size and foam 512 stability. International Journal of Mineral Processing 64, 69-80. 
513 Crowe, C. T., Schwarzkopf, J. D., Sommerfeld, M. and Tsuji, Y., 2011. Multiphase flows with 514 droplets and particles, second edition. CRC Press.

515 Dai, Z., Dukhin, S., Fornasiero, D. and Ralston, J., 1998. The inertial hydrodynamic interaction 516 of particles and rising bubbles with mobile surfaces. J Colloid Interface Sci 197, 275-292.

517 Das, S. C., Larson, I., Morton, D. A. and Stewart, P. J., 2011. Determination of the polar and total 518 surface energy distributions of particulates by inverse gas chromatography. Langmuir 27, 521519523

520 Davies, G. B., Kruger, T., Coveney, P. V. and Harting, J., 2014. Detachment energies of spheroidal particles from fluid-fluid interfaces. J Chem Phys 141, 154902.

Forssberg, K. S. E., Subrahmanyam, T. V. and Nilsson, L. K., 1993. Influence of grinding method on complex sulphide ore flotation: A pilot plant study. International Journal of Mineral Processing 38, 157-175.

Fuerstenau, M. C., Jameson, G. J. and Yoon, R.-H., 2007. Froth flotation: A century of innovation. Society for Mining, Metallurgy and Exploration, Inc. (SME).

Gamble, J. F., Leane, M., Olusanmi, D., Tobyn, M., Supuk, E., Khoo, J. and Naderi, M., 2012.

528 Surface energy analysis as a tool to probe the surface energy characteristics of micronized materials-a comparison with inverse gas chromatography. International Journal of Pharmaceutics 422, 238-244.

531 Gu, G., Sean Sanders, R., Nandakumar, K., Xu, Z. and Masliyah, J. H., 2004. A novel 532 experimental technique to study single bubble-bitumen attachment in flotation. International Journal of Mineral Processing 74, 15-29.

Huang, Z., Legendre, D. and Guiraud, P., 2011. A new experimental method for determining particle capture efficiency in flotation. Chemical Engineering Science 66, 982-997.

Huang, Z., Legendre, D. and Guiraud, P., 2012. Effect of interface contamination on particlebubble collision. Chemical Engineering Science 68, 1-18.

538 Hubička, M., Basařová, P. and Vejražka, J., 2013. Collision of a small rising bubble with a large 539 falling particle. International Journal of Mineral Processing 121, 21-30.

540 Jameson, G. J., 2010. Advances in fine and coarse particle flotation. Canadian Metallurgical 541 Quarterly 49, 325-330.

542 Kasper, G., Niida, T. and Yang, M., 1985. Measurements of viscous drag on cylinders and chains 543 of spheres with aspect ratios between 2 and 50. Journal of Aerosol Science 16, 535-556. 
544 Kemper, M., 1999. State-of-the-art and new technologies in flotation deinking. International $545 \quad$ Journal of Mineral Processing 56, 317-333.

546 Koh, P. T. L., Hao, F. P., Smith, L. K., Chau, T. T. and Bruckard, W. J., 2009. The effect of

547 particle shape and hydrophobicity in flotation. International Journal of Mineral Processing 93, $548 \quad 128-134$.

549 Kursun, H. and Ulusoy, U., 2006. Influence of shape characteristics of talc mineral on the column 550 flotation behavior. International Journal of Mineral Processing 78, 262-268.

551 Lecrivain, G., Barry, L. and Hampel, U., 2014. Three-dimensional simulation of multilayer 552 particle deposition in an obstructed channel flow. Powder Technology 258, 134-143.

553 Lecrivain, G. and Hampel, U., 2012. Influence of the lagrangian integral time scale estimation in 554 the near wall region on particle deposition. ASME Journal of Fluids Engineering 134, 1-6.

555 Legendre, D., Sarrot, V. and Guiraud, P., 2009. On the particle inertia-free collision with a 556 partially contaminated spherical bubble. International Journal of Multiphase Flow 35, 163170.

558 Loth, E., 2008. Drag of non-spherical solid particles of regular and irregular shape. Powder 559 Technology 182, 342-353.

560 Mohammadi-Jam, S. and Waters, K. E., 2014. Inverse gas chromatography applications: A 561 review. Adv Colloid Interface Sci 212, 21-44.

562 Nguyen, A. V., 2003. New method and equations for determining attachment tenacity and 563 particle size limit in flotation. International Journal of Mineral Processing 68, 167-182.

564 Nguyen, A. V. and Jameson, G. J., 2005. Sliding of fine particles on the slip surface of rising gas 565 bubbles: Resistance of liquid shear flows. International Journal of Multiphase Flow 31, 492566513.

567 Nguyen, P. T. and Nguyen, A. V., 2009. Validation of the generalised sutherland equation for 568 bubble-particle encounter efficiency in flotation: Effect of particle density. Minerals 569 Engineering 22, 176-181.

570 Nowak, E., Robbins, P., Combes, G., Stitt, E. H. and Pacek, A. W., 2013. Measurements of 571 contact angle between fine, non-porous particles with varying hydrophobicity and water and non-polar liquids of different viscosities. Powder Technology 250, 21-32.

$574 \quad$ Chemical Engineering Science 12, 133-141. 
575 Oberbeck, A., 1876. Über stationäre flüssigkeitsbewegungen mit berücksichtigung der inneren 576 reibung. Journal für die reine und angewandte Mathematik 81, 62-81.

577 Rahimi, M., Dehghani, F., Rezai, B. and Aslani, M. R., 2012. Influence of the roughness and 578 shape of quartz particles on their flotation kinetics. International Journal of Minerals $579 \quad$ Metallurgy and Materials 19, 284-289.

580 Ralston, J., Fornasiero, D. and Hayes, R., 1999. Bubble-particle attachment and detachment in 581 flotation. International Journal of Mineral Processing 56, 133-164.

Ren, Y., Wu, Z., Ondruschka, B., Braeutigam, P., Franke, M., Nehring, H. and Hampel, U., 2011. Oxidation of phenol by microbubble-assisted microelectrolysis. Chemical Engineering \& Technology 34, 699-706.

Rosenqvist, T., 2004. Principles of extractive metallurgy. Tapir Academic Press.

Rubio, J., Souza, M. L. and Smith, R. W., 2002. Overview of flotation as a wastewater treatment technique. Minerals Engineering 15, 139-155.

Sarrot, V., Guiraud, P. and Legendre, D., 2005. Determination of the collision frequency between bubbles and particles in flotation. Chemical Engineering Science 60, 6107-6117.

Schindelin, J., Arganda-Carreras, I., Frise, E., Kaynig, V., Longair, M., Pietzsch, T., Preibisch,

591 S., Rueden, C., Saalfeld, S., Schmid, B., Tinevez, J.-Y., White, D. J., Hartenstein, V., Eliceiri, K., Tomancak, P. and Cardona, A., 2012. Fiji: An open-source platform for biological-image analysis. Nature Methods 9, 676-682.

Schulze, H. J., 1989. Hydrodynamics of bubble-mineral particle collisions. Mineral Processing and Extractive Metallurgy Review 5, 43-76.

Schulze, H. J., 1992. Probability of particle attachment on gas bubbles by sliding. Advances in Colloid and Interface Science 40, 283-305.

Susana, L., Campaci, F. and Santomaso, A. C., 2012. Wettability of mineral and metallic powders: Applicability and limitations of sessile drop method and washburn's technique. Powder Technology 226, 68-77.

Tao, D., 2005. Role of bubble size in flotation of coarse and fine particles-a review. Separation Science and Technology 39, 741-760.

604 contact angle measurements. Journal of Colloid and Interface Science 179, 635-636.

605 van Oss, C. J., 2003. Long-range and short-range mechanisms of hydrophobic attraction and 606 hydrophilic repulsion in specific and aspecific interactions. J Mol Recognit 16, 177-190. 
607 van Oss, C. J., Giese, R. F. and Docoslis, A., 2005. Hyperhydrophobicity of the water-air 608 interface. Journal of Dispersion Science and Technology 26, 585-590.

609 VDI-GVC, 2013. Verein Deutscher Ingenieure (VDI) - Gesellschaft Verfahrenstechnik und 610 Chemieingenieurwesen (GVC), Wärmeatlas, $11^{\text {th }}$ Edition. VDI-Verlag, Springer.

611 Verrelli, D. I., Bruckard, W. J., Koh, P. T. L., Schwarz, M. P. and Follink, B., 2014. Particle

612 shape effects in flotation. Part 1: Microscale experimental observations. Minerals Engineering $61358,80-89$.

614 Verrelli, D. I., Koh, P. T. L., Bruckard, W. J. and Schwarz, M. P., 2012. Variations in the 615 induction period for particle-bubble attachment. Minerals Engineering 36-38, 219-230.

616 Verrelli, D. I., Koh, P. T. L. and Nguyen, A. V., 2011. Particle-bubble interaction and attachment 617 in flotation. Chemical Engineering Science 66, 5910-5921.

618 Wang, W., Zhou, Z., Nandakumar, K., Xu, Z. and Masliyah, J. H., 2003a. Attachment of 619 individual particles to a stationary air bubble in model systems. International Journal of $620 \quad$ Mineral Processing 68, 47-69.

621 Wang, W., Zhou, Z., Nandakumar, K., Xu, Z. and Masliyah, J. H., 2003b. Effect of surface 622 mobility on the particle sliding along a bubble or a solid sphere. Journal of Colloid and 623 Interface Science 259, 81-88.

624 Ye, Y., Khandrika, S. M. and Miller, J. D., 1989. Induction-time measurements at a particle bed. 625 International Journal of Mineral Processing 25, 221-240.

626 Yekeler, M., Ulusoy, U. and Hiçyılmaz, C., 2004. Effect of particle shape and roughness of talc 627 mineral ground by different mills on the wettability and floatability. Powder Technology 140, $628 \quad 68-78$.

629 Yoon, R. H. and Luttrell, G. H., 1989. The effect of bubble size on fine particle flotation. Mineral 630 Processing and Extractive Metallurgy Review 5, 101-122. 


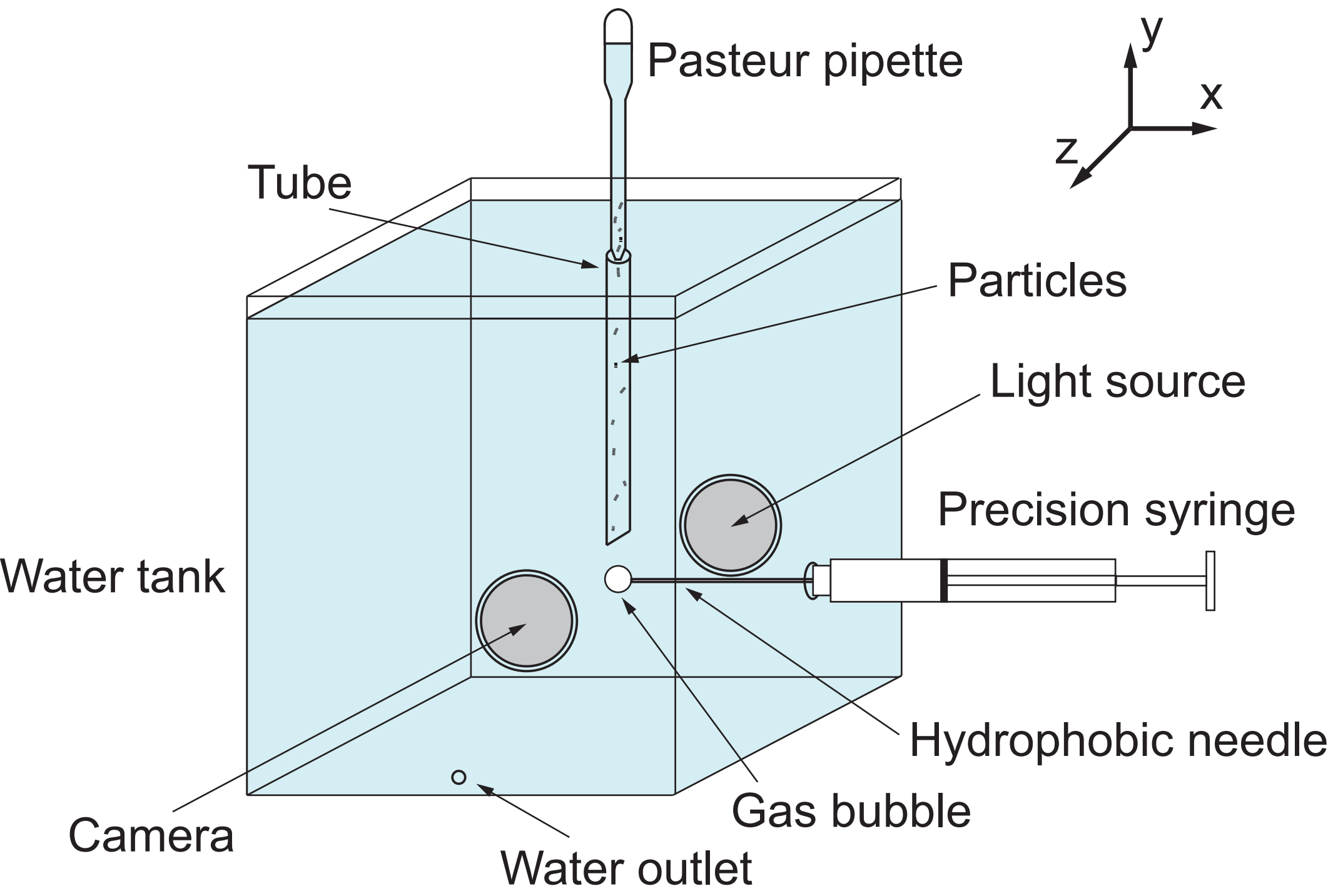




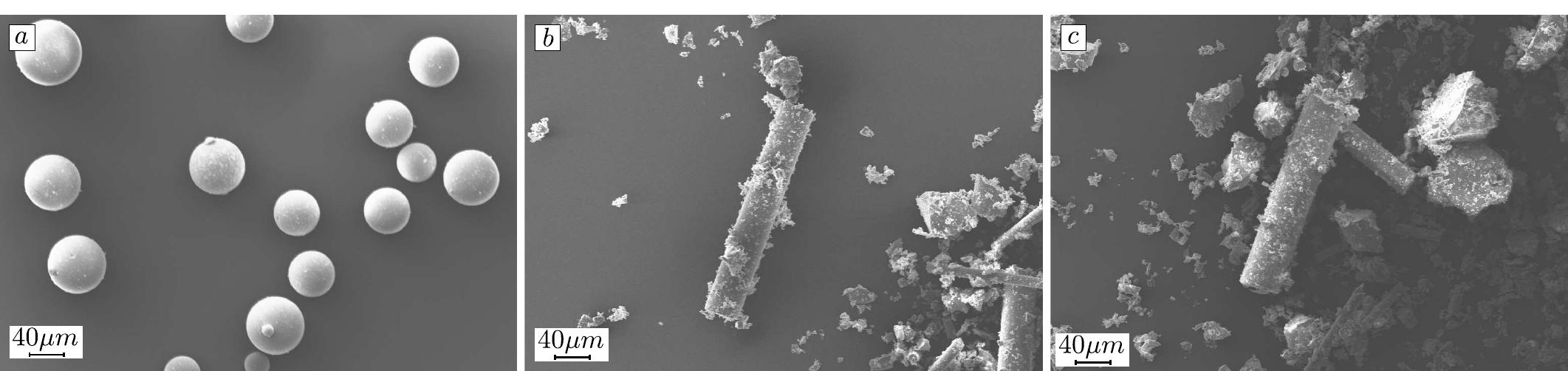




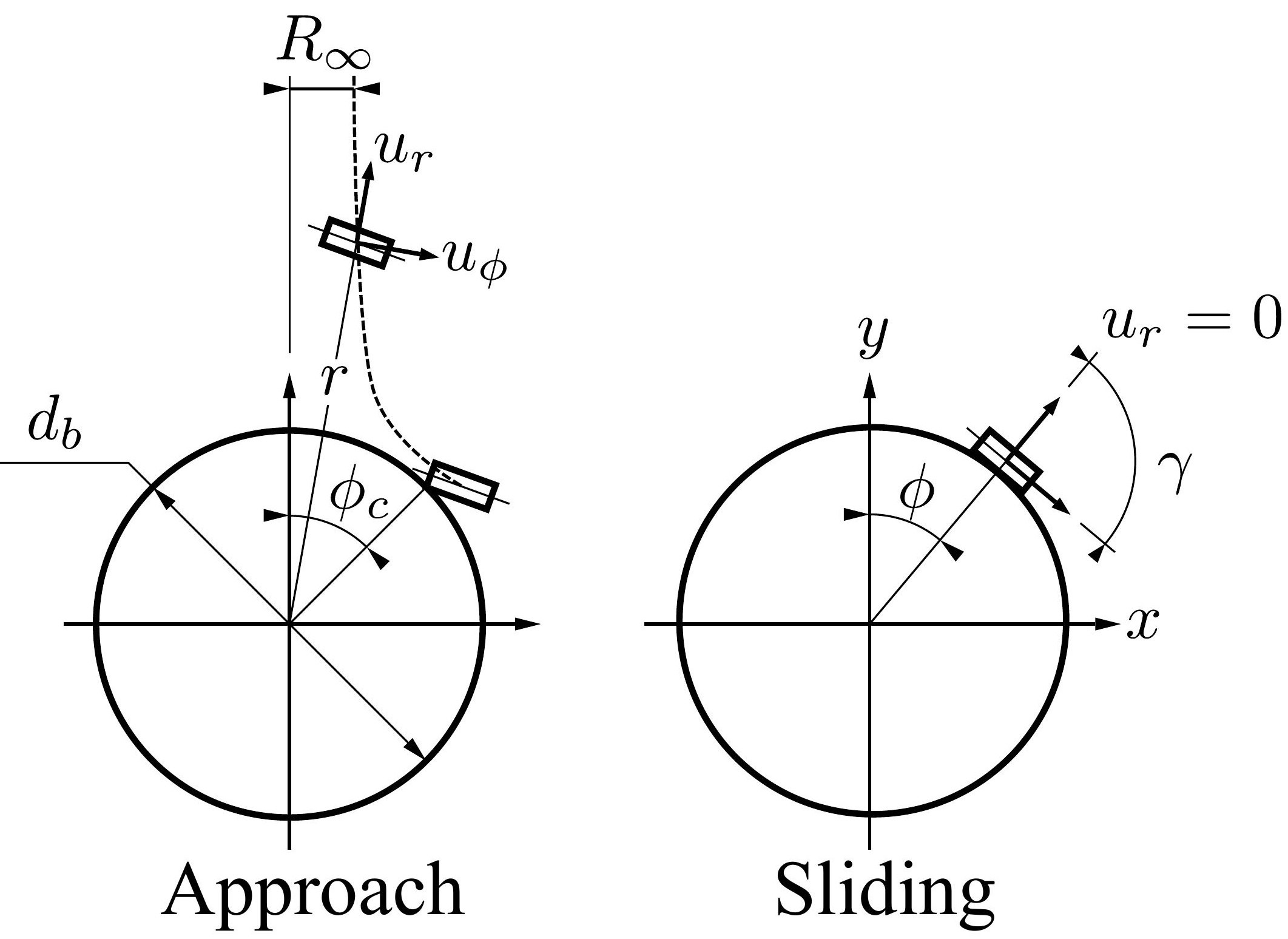

\section{Sliding}



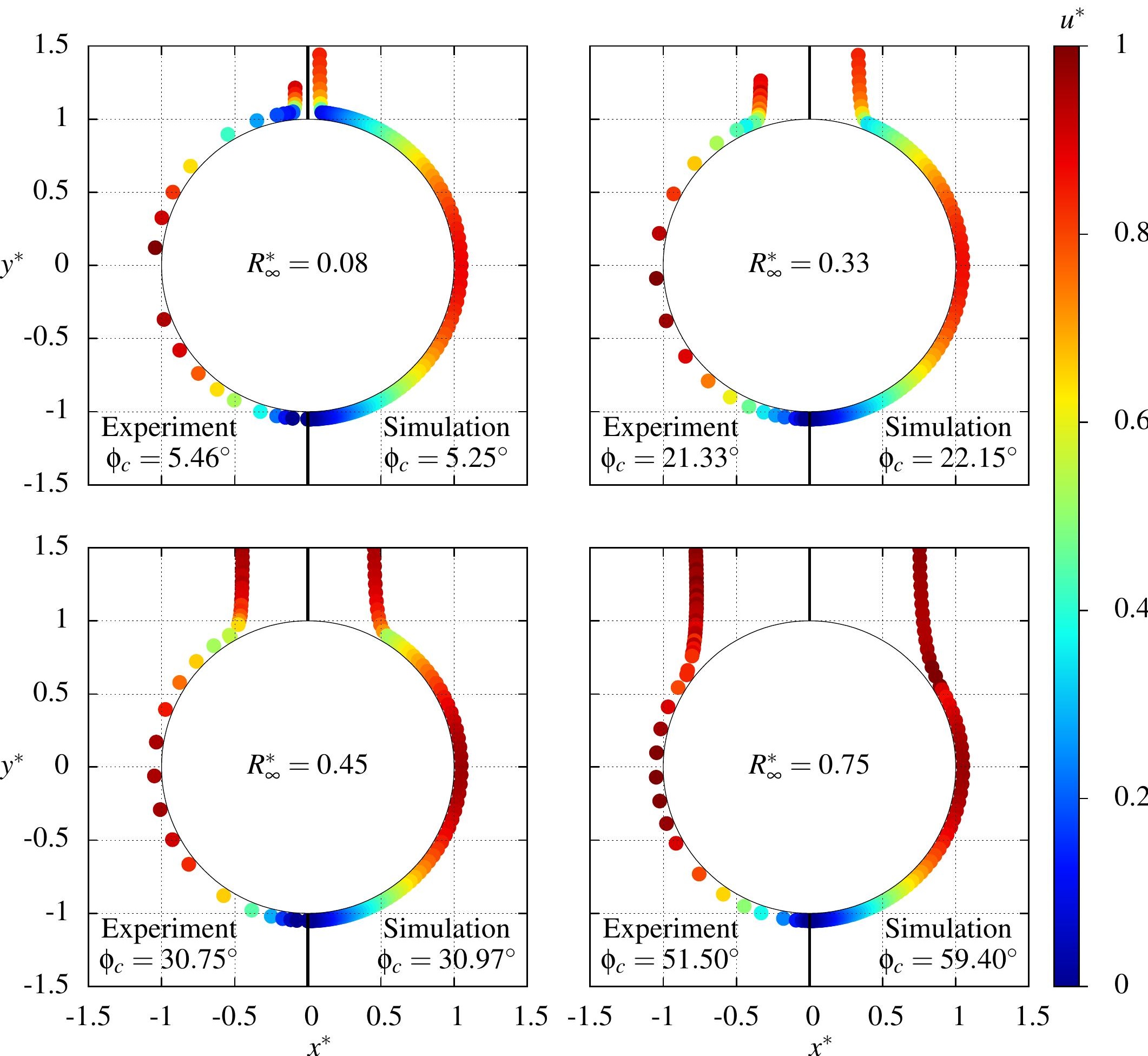

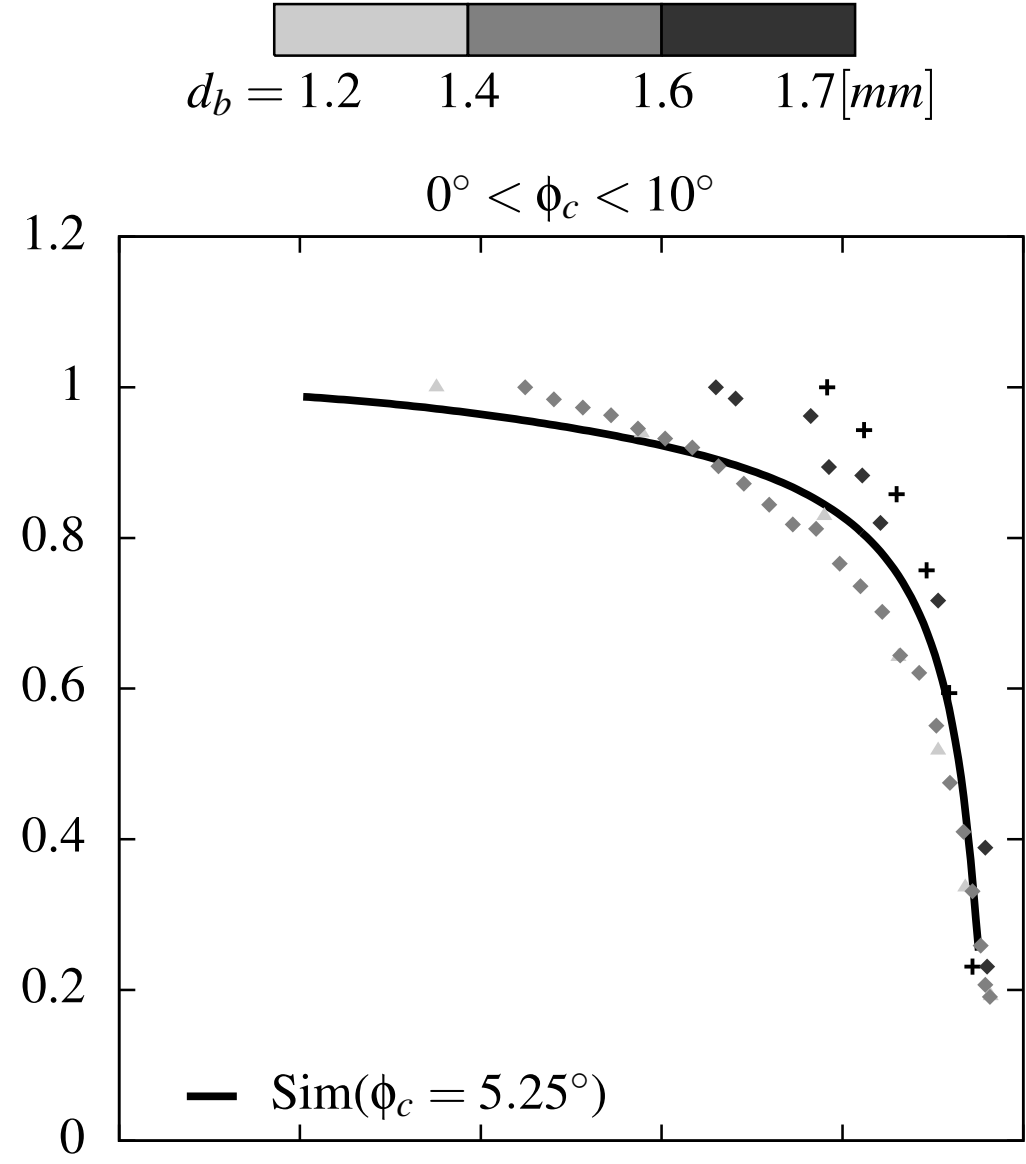

$1.230^{\circ}<\phi_{c}<50^{\circ}$

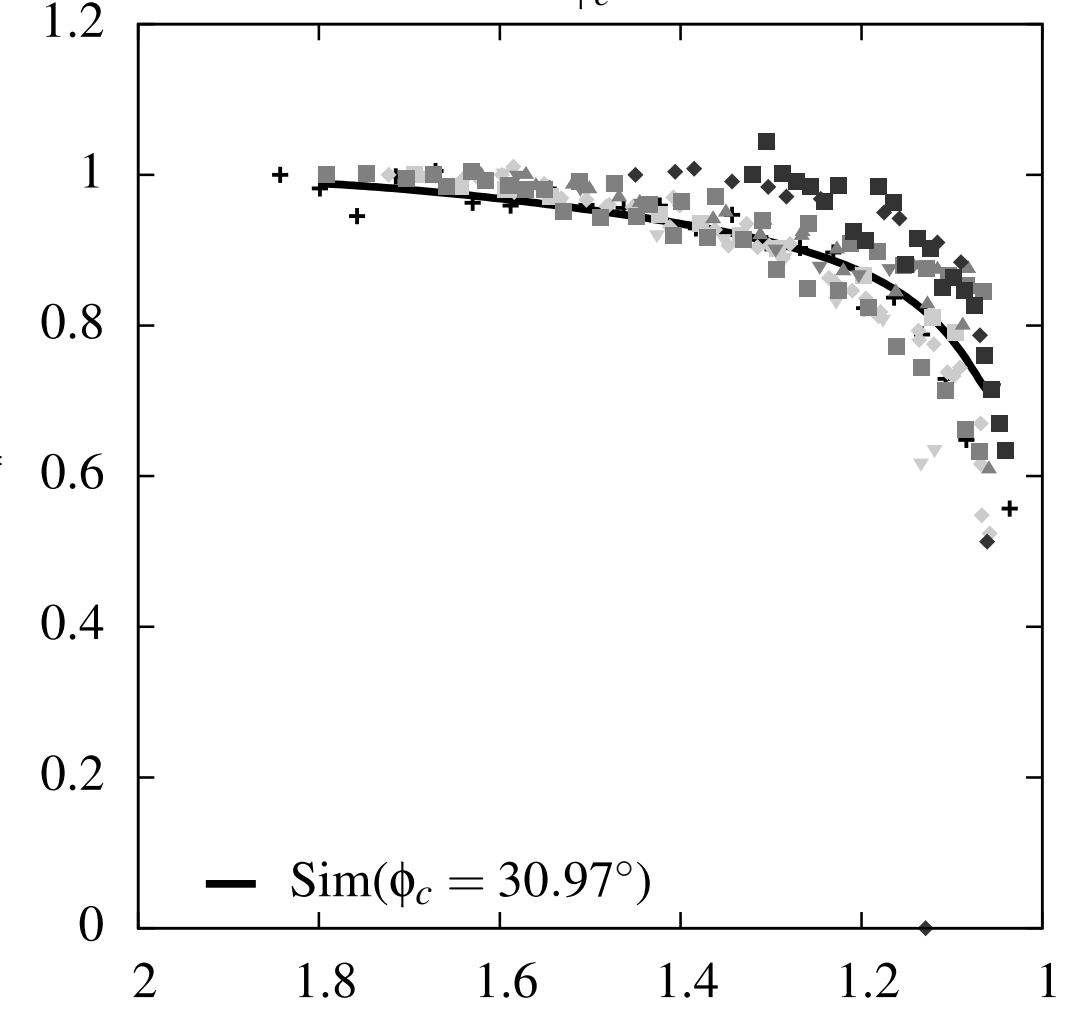

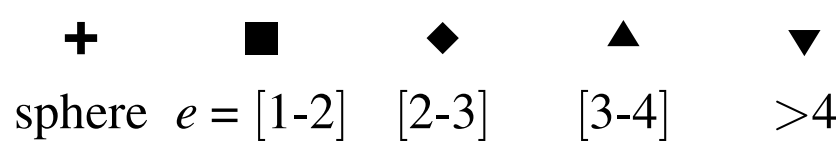
$10^{\circ}<\phi_{c}<30^{\circ}$

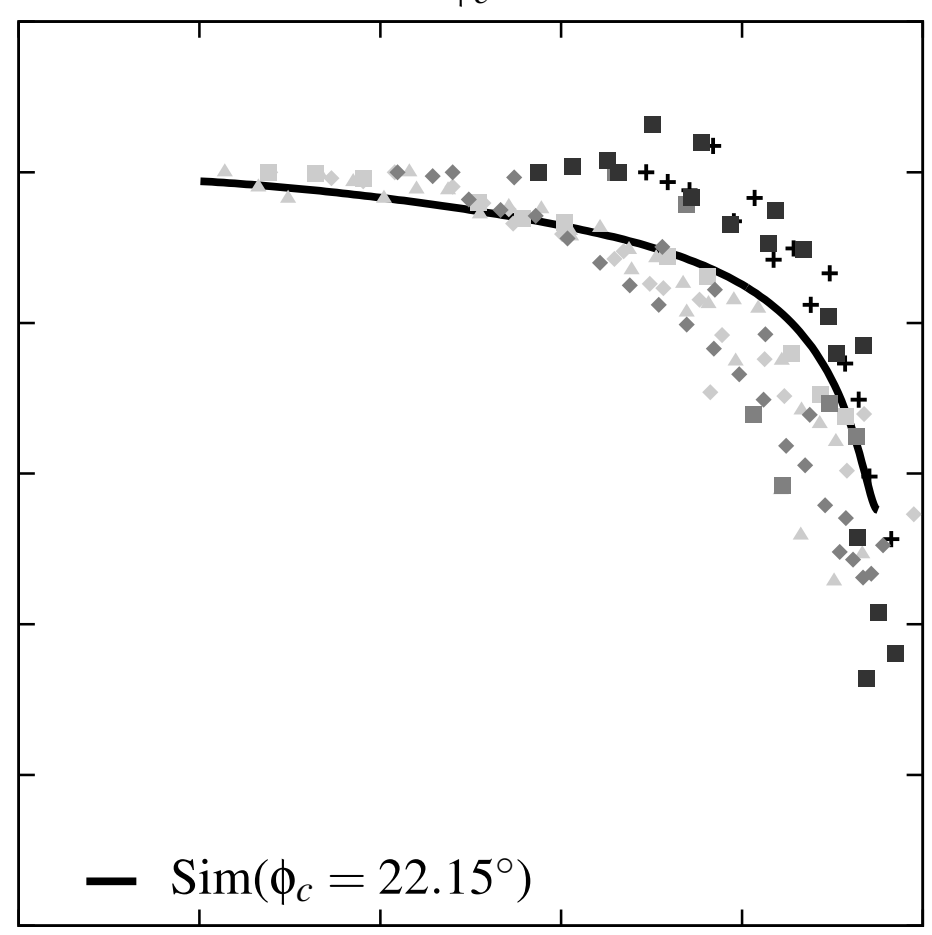

$\phi_{c}>50^{\circ}$

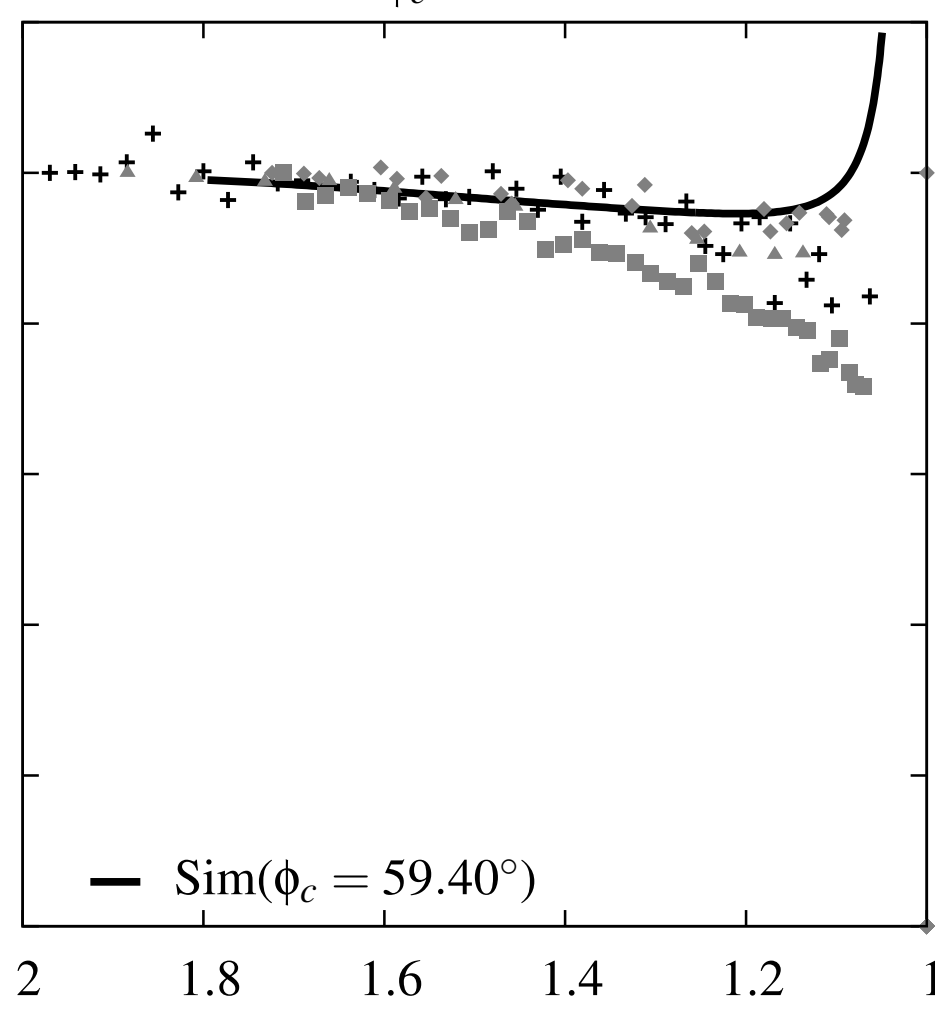
$r^{\pi}$ 

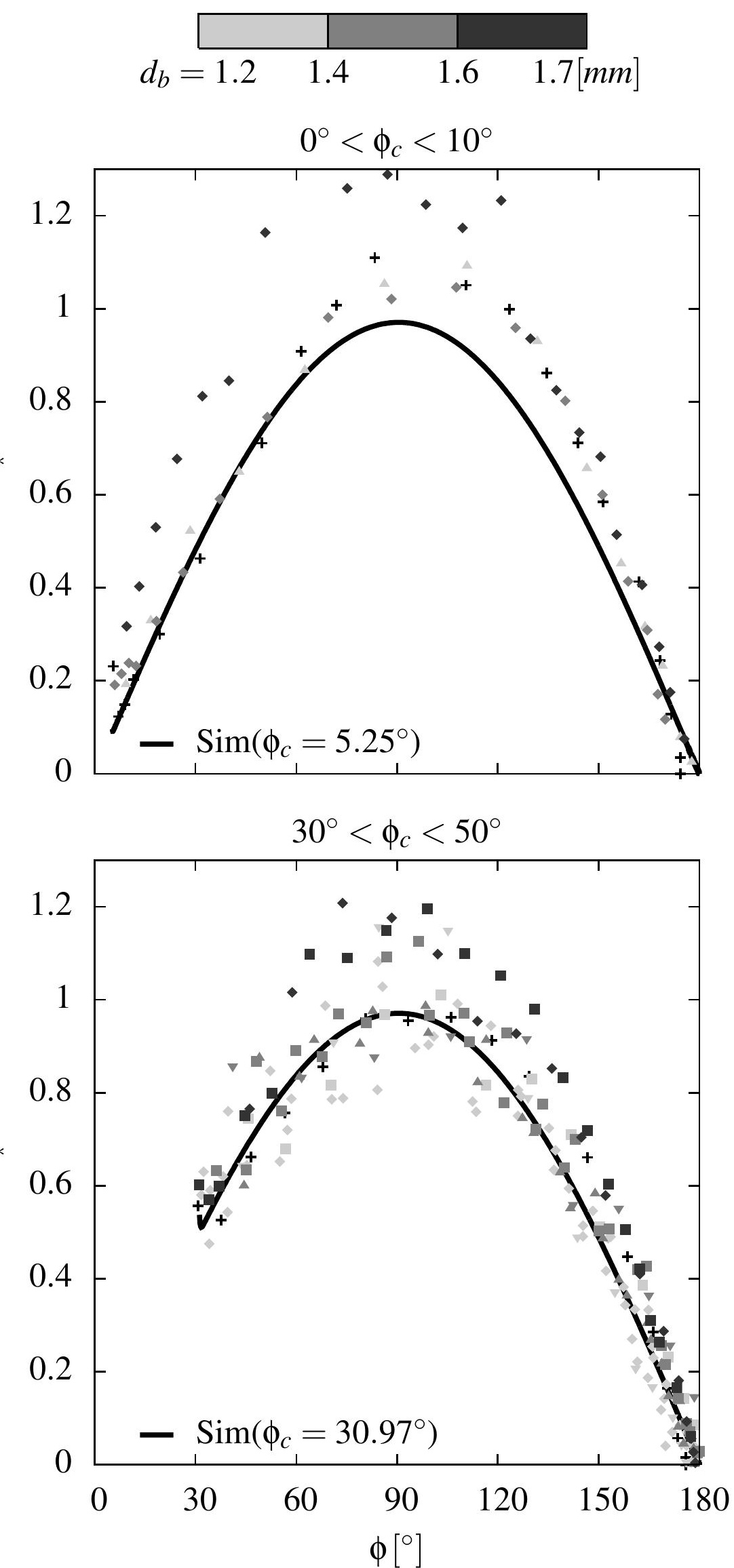
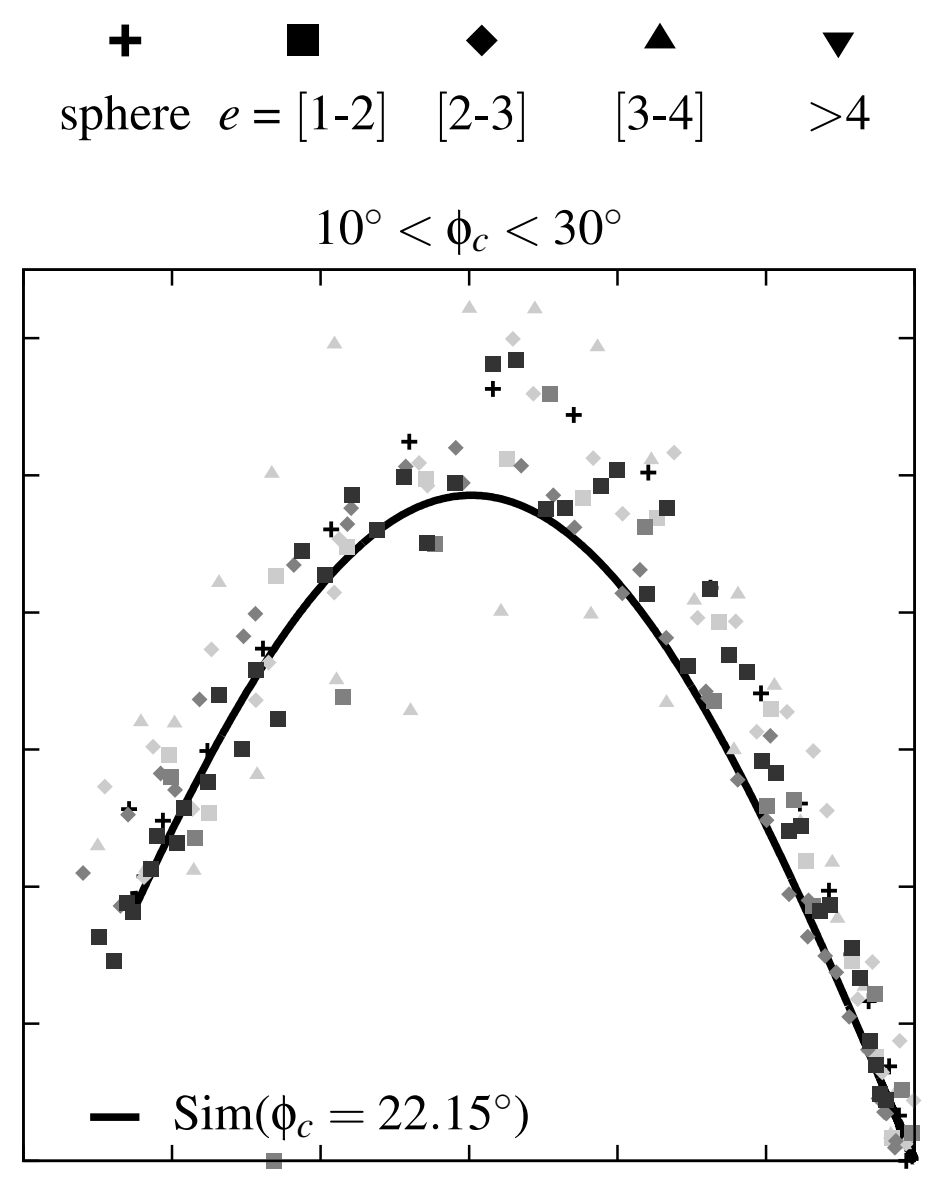

$\phi_{c}>50^{\circ}$

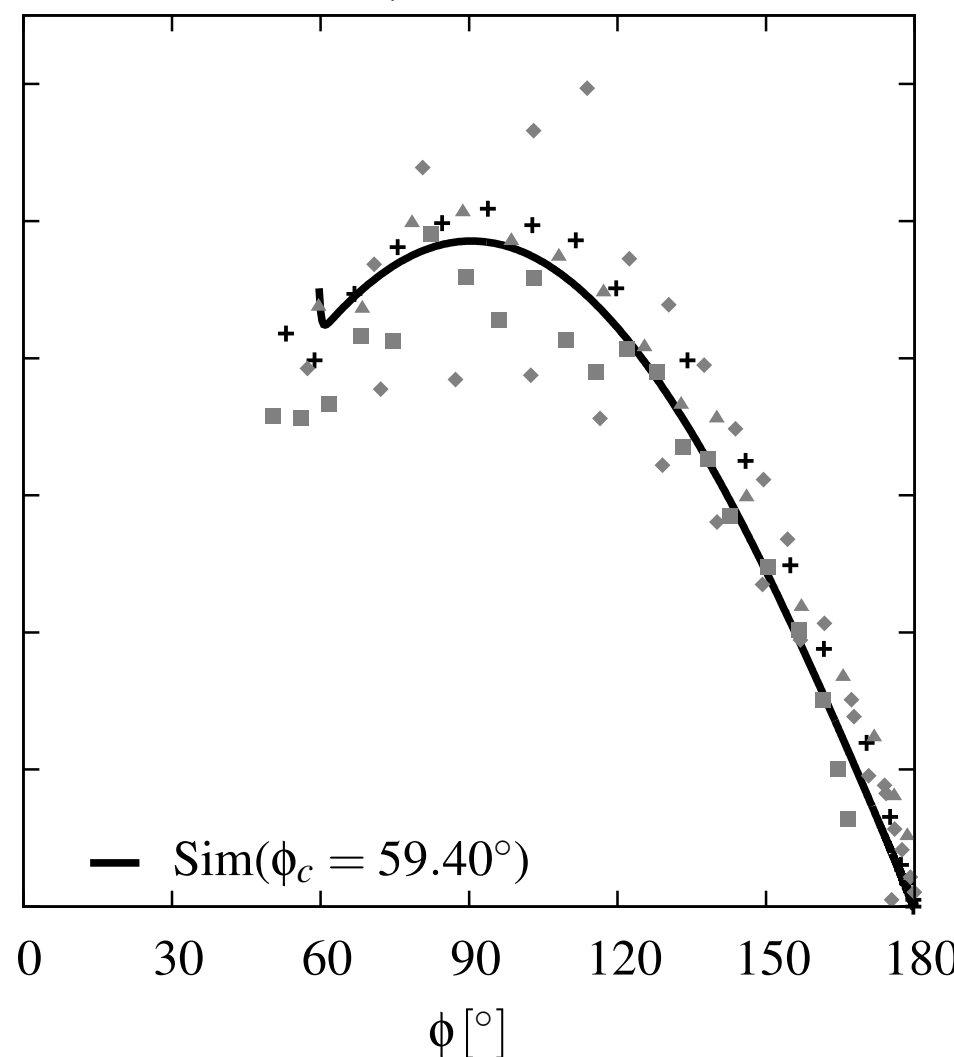



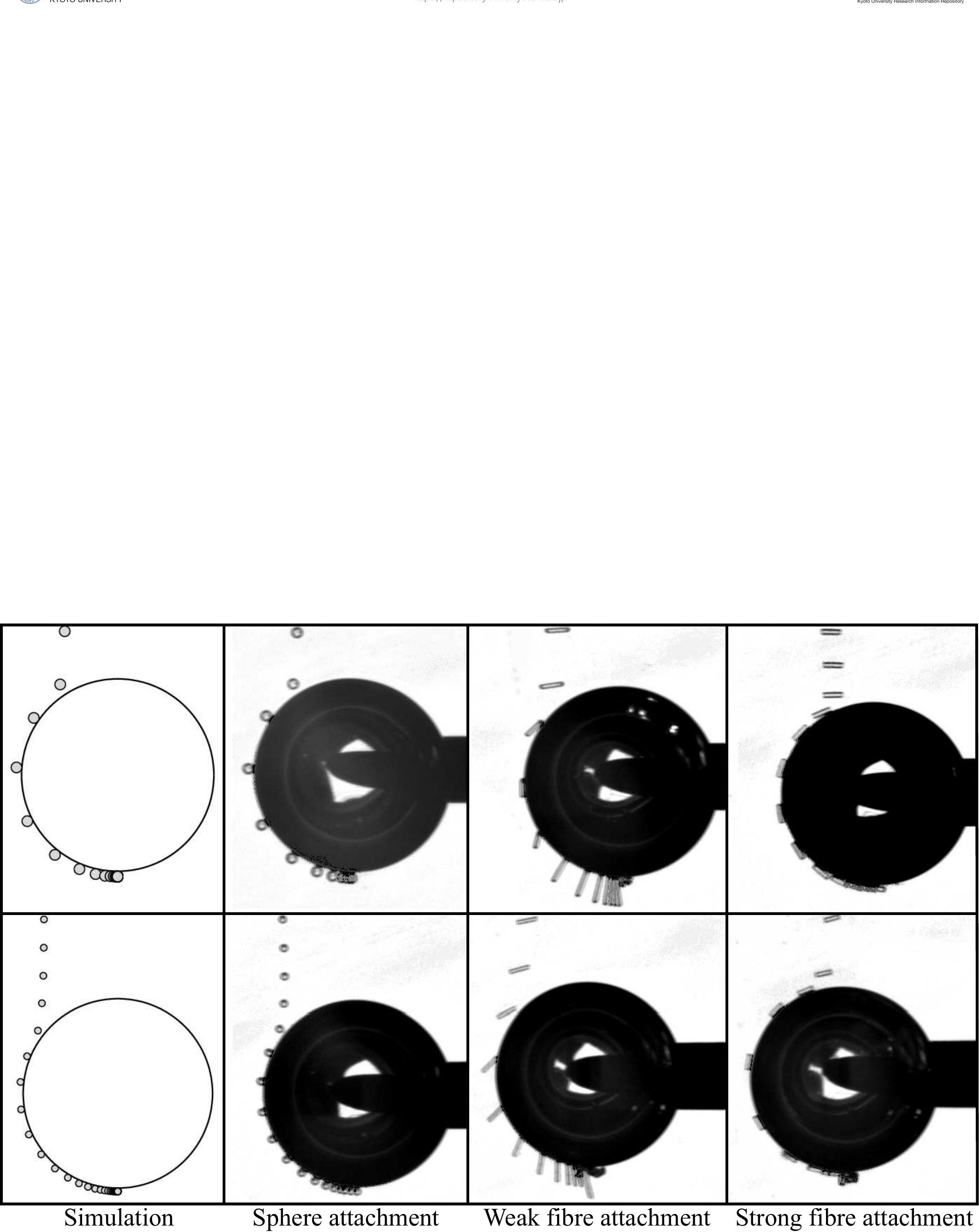

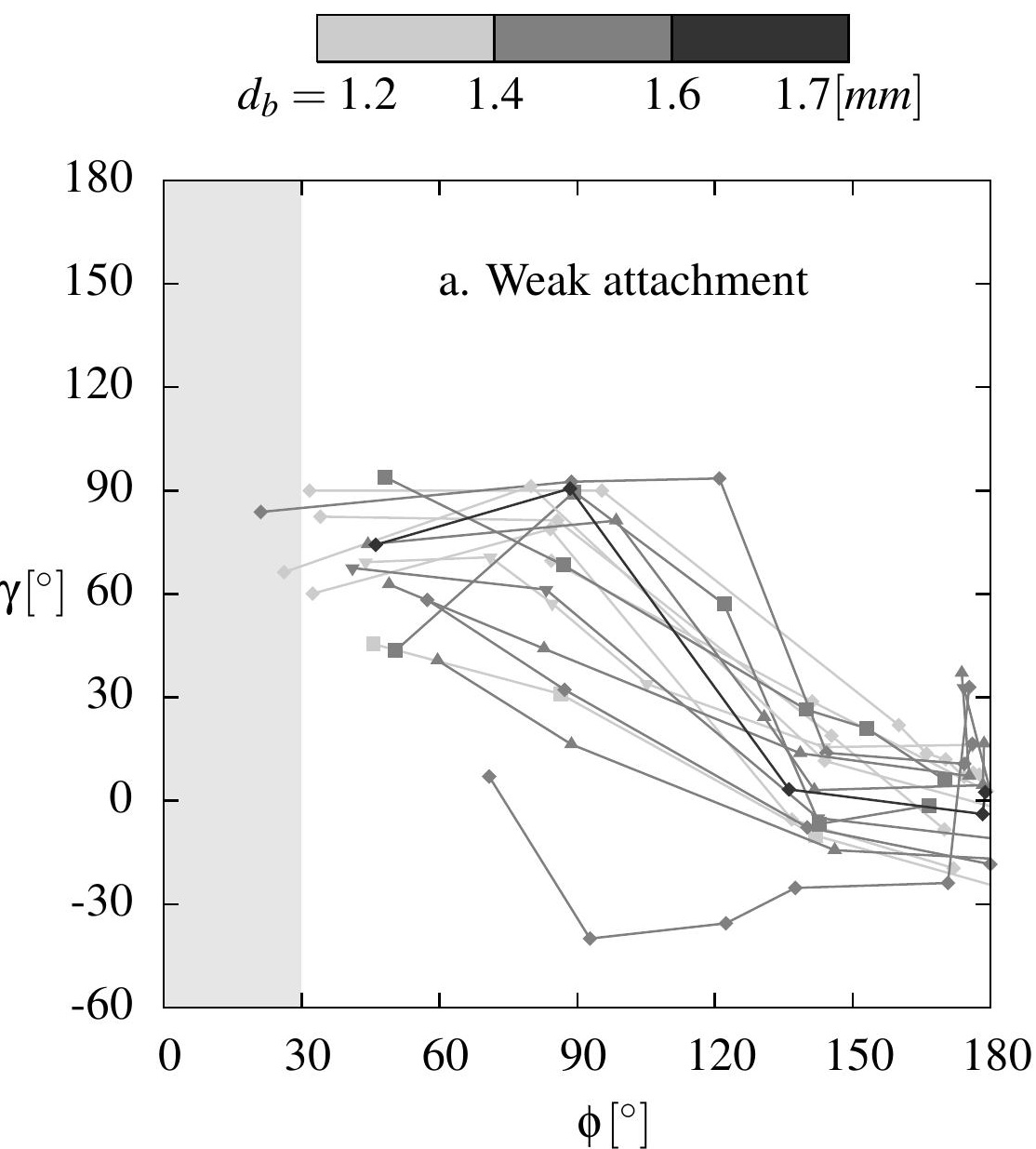
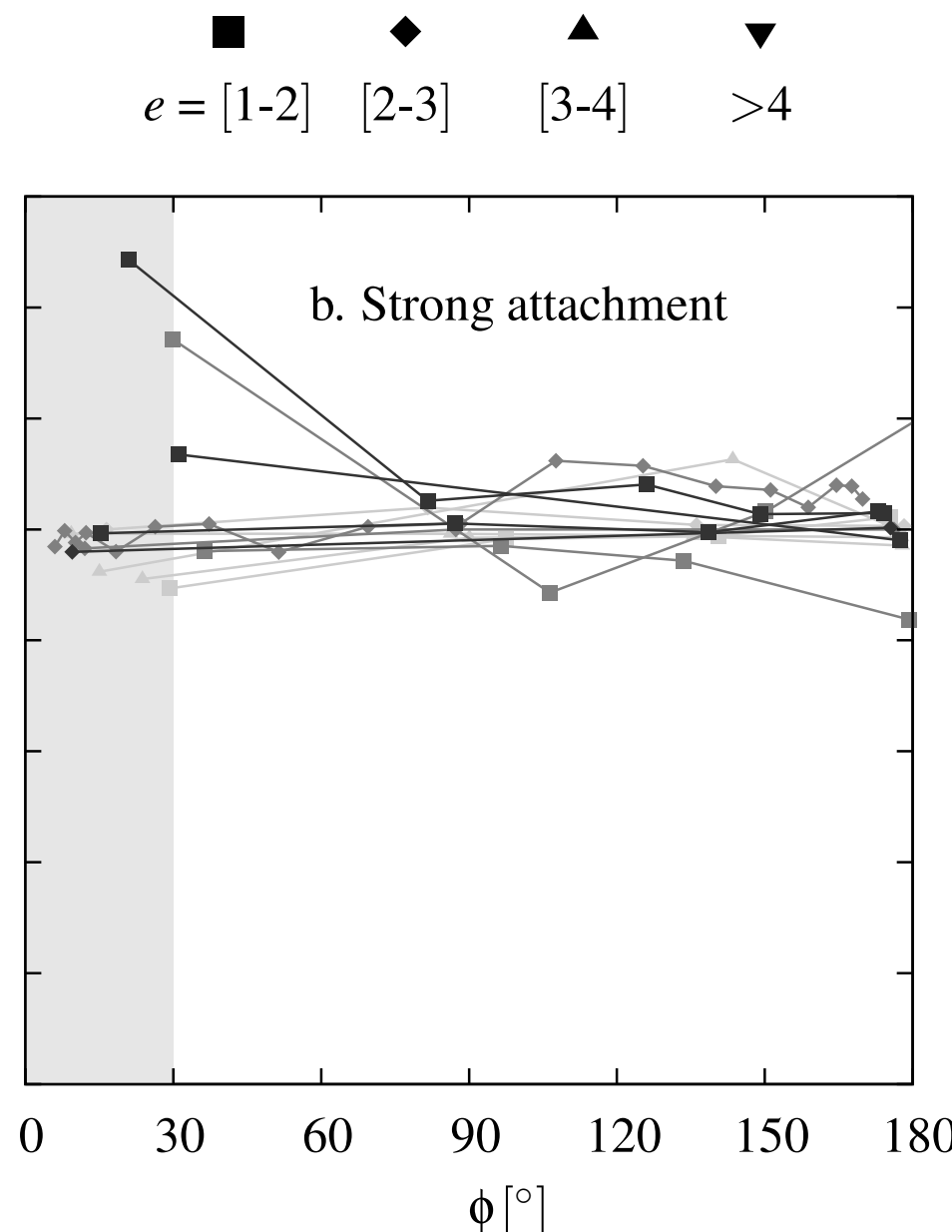


\begin{tabular}{|c|c|c|c|c|c|c|c|c|}
\hline material & $\begin{array}{c}S_{m}^{B E T} \\
{\left[\mathrm{~m}^{2} / \mathrm{g}\right]}\end{array}$ & $\begin{array}{c}\text { surface } \\
\text { coverage }\end{array}$ & $\begin{array}{c}\gamma^{d} \\
{\left[\mathrm{~mJ} / \mathrm{m}^{2}\right]}\end{array}$ & $\begin{array}{c}\gamma^{-} \\
{\left[\mathrm{mJ} / \mathrm{m}^{2}\right]}\end{array}$ & $\begin{array}{c}\gamma^{+} \\
{\left[\mathrm{mJ} / \mathrm{m}^{2}\right]}\end{array}$ & $\begin{array}{c}\gamma^{+} \\
{\left[\mathrm{mJ} / \mathrm{m}^{2}\right]}\end{array}$ & $\begin{array}{c}\Delta G_{p w b} \\
{\left[\mathrm{~mJ} / \mathrm{m}^{2}\right]}\end{array}$ & $\begin{array}{c}\theta \\
{\left[{ }^{\circ}\right]}\end{array}$ \\
\hline Liquid water & - & - & 18.0 & 21.1 & 21.1 & 60.2 & - & - \\
\hline Gas bubble & - & - & 0.0 & 0.0 & 0.0 & 0.0 & - & - \\
\hline \multirow{3}{*}{ spherical particles } & \multirow{3}{*}{0.069} & $1 \%$ & $\begin{array}{r}47.6 \\
\pm 3.5 \\
\end{array}$ & $\begin{array}{c}3.5 \\
\pm 1.2 \\
\end{array}$ & $\begin{array}{c}1.6 \\
\pm 0.3 \\
\end{array}$ & $\begin{array}{l}52.2 \\
\pm 4.8 \\
\end{array}$ & $\begin{array}{l}-33.3 \\
\pm 9.0 \\
\end{array}$ & $\begin{array}{r}63.5 \\
\pm 6.7 \\
\end{array}$ \\
\hline & & $10 \%$ & $\begin{array}{l}49.3 \\
\pm 3.3\end{array}$ & $\begin{array}{c}3.8 \\
\pm 1.2\end{array}$ & $\begin{array}{c}0.9 \\
\pm 0.2\end{array}$ & $\begin{array}{l}53.1 \\
\pm 4.4\end{array}$ & $\begin{array}{l}-34.0 \\
\pm 8.8\end{array}$ & $\begin{array}{l}64.2 \\
\pm 6.3\end{array}$ \\
\hline & & $100 \%$ & $\begin{array}{r}49.3 \\
\pm 3.4 \\
\end{array}$ & $\begin{array}{c}3.5 \\
\pm 1.0 \\
\end{array}$ & $\begin{array}{c}0.5 \\
\pm 0.2 \\
\end{array}$ & $\begin{array}{l}52.1 \\
\pm 4.2 \\
\end{array}$ & $\begin{array}{l}-36.8 \\
\pm 8.7 \\
\end{array}$ & $\begin{array}{r}67.2 \\
\pm 5.8 \\
\end{array}$ \\
\hline \multirow{3}{*}{ elongated particles } & \multirow{3}{*}{0.470} & $1 \%$ & $\begin{array}{l}37.4 \\
\pm 0.3 \\
\end{array}$ & $\begin{array}{c}4.6 \\
\pm 0.1 \\
\end{array}$ & $\begin{array}{c}4.0 \\
\pm 0.0\end{array}$ & $\begin{array}{l}46.0 \\
\pm 0.4 \\
\end{array}$ & $\begin{array}{l}-30.3 \\
\pm 3.4 \\
\end{array}$ & $\begin{array}{l}60.2 \\
\pm 0.6 \\
\end{array}$ \\
\hline & & $10 \%$ & $\begin{array}{l}40.8 \\
\pm 0.6\end{array}$ & $\begin{array}{c}3.3 \\
\pm 0.2\end{array}$ & $\begin{array}{c}1.0 \\
\pm 0.1\end{array}$ & $\begin{array}{l}44.5 \\
\pm 0.8\end{array}$ & $\begin{array}{l}-40.0 \\
\pm 4.0 \\
\end{array}$ & $\begin{array}{r}70.4 \\
\pm 1.2 \\
\end{array}$ \\
\hline & & $100 \%$ & $\begin{array}{l}40.8 \\
\pm 0.9\end{array}$ & $\begin{array}{c}3.0 \\
\pm 0.2\end{array}$ & $\begin{array}{c}1.1 \\
\pm 0.1\end{array}$ & $\begin{array}{l}44.4 \\
\pm 1.1\end{array}$ & $\begin{array}{l}-40.7 \\
\pm 4.4\end{array}$ & $\begin{array}{l}71.1 \\
\pm 1.4\end{array}$ \\
\hline
\end{tabular}




\begin{tabular}{|l|c|c|}
\cline { 2 - 3 } \multicolumn{1}{c|}{} & $\kappa_{\|}$ & $\kappa_{\perp}$ \\
\hline $\begin{array}{c}\text { Approximation } \\
(1<e<6)\end{array}$ & $\left(\frac{4}{5}+\frac{e}{5}\right) e^{-1 / 3}$ & $\left(\frac{3}{5}+\frac{2 e}{5}\right) e^{-1 / 3}$ \\
\hline$e=3$ (Approximation1) & 0.97 & 1.25 \\
\hline$e=3$ (Experiment2) & 1.06 & 1.26 \\
\hline & & \\
\hline$e=6$ (Approximation1) & 1.10 & 1.65 \\
\hline$e=6$ (Experiment2) & 1.20 & 1.52 \\
\hline
\end{tabular}




\section{Figure captions:}

Figure 1: Schematic of the test facility.

Figure 2: Images of the spherical (a) and elongated particles (b, c) obtained with scanning electron microscope.

Figure 3: Schematic representation of an elongated particle in its polar system during the approach and the sliding phase.

Figure 4: Radial and tangential drag correction factors shown as a function of the gap.

Figure 5: Validation of simulated trajectories coloured by velocity magnitude. Simulations and experiments were performed with spherical particles.

Figure 6: Evolution of the particle velocity magnitude during the approach phase. The data are sorted by collision angles.

Figure 7: Evolution of the particle velocity magnitude during the sliding phase. The data are sorted by collision angles.

Figure 8: Simulated attachment of a spherical particle, experimental attachment of a spherical particle, experimental weak attachment of an elongated particle and experimental strong attachment of an elongated particle (from left to right).

Figure 9: About 90\% of experimental runs, in which the collision angle exceeded the threshold $\phi_{c}>30^{\circ}$, resulted in a weak attachment (Subfigure a). Should the collision angle be lower than this threshold collision angle (grey area in Subfigure b), the attachment takes a strong form.

\section{Table captions:}

Table 1: Measured hydrophobicity of the spherical particles and of the elongated particles at surface coverages of $1 \%, 10 \%$ and $100 \%$.

Table 2: Estimations of the two shape factors of an elongated particle. The approximation is derived from the work of Loth (2008). The experimental values are measured from a falling chain of beads (Kasper, Niida et al., 1985). 


\begin{tabular}{|c|c|c|c|c|c|c|c|}
\hline & $\begin{array}{l}\text { Particle } \\
\text { ID }\end{array}$ & $\begin{array}{c}e \\
{[-]}\end{array}$ & $\begin{array}{c}\text { major axis } \\
\text { length } \\
{[\mu \mathrm{m}]}\end{array}$ & $\begin{array}{c}u_{\infty} \\
{[\mathrm{mm} / \mathrm{s}]}\end{array}$ & $\begin{array}{c}R_{\infty}^{*} \\
{[-]}\end{array}$ & $\begin{array}{l}\phi_{c} \\
{\left[{ }^{\circ}\right]}\end{array}$ & $\begin{array}{c}d_{b} \\
{[\mu \mathrm{m}]}\end{array}$ \\
\hline \multirow{30}{*}{$\begin{array}{c}\text { elongated } \\
\text { particles } \\
\text { (Experiment) }\end{array}$} & 1 & 3.5 & 181 & 3.00 & 0.47 & 23.72 & 1363.1 \\
\hline & 2 & 2.0 & 143 & 6.80 & 0.47 & 31.74 & 1378.2 \\
\hline & 3 & 2.5 & 160 & 5.50 & 0.13 & 16.42 & 1378.2 \\
\hline & 4 & 2.2 & 155 & 6.80 & 0.54 & 39.70 & 1372.8 \\
\hline & 5 & 3.6 & 215 & 6.50 & 0.27 & 15.03 & 1378.2 \\
\hline & 6 & 4.8 & 285 & 8.50 & 0.70 & 43.95 & 1378.2 \\
\hline & 7 & 1.7 & 106 & 4.50 & 0.70 & 45.63 & 1316.7 \\
\hline & 8 & 1.9 & 118 & 4.30 & 0.40 & 29.35 & 1306.3 \\
\hline & 9 & 2.3 & 144 & 4.63 & 0.57 & 32.42 & 1345.0 \\
\hline & 10 & 2.5 & 164 & 4.57 & 0.49 & 34.11 & 1345.0 \\
\hline & 11 & 3.5 & 227 & 5.90 & 0.21 & 9.27 & 1265.5 \\
\hline & 12 & 2.4 & 148 & 5.70 & 0.71 & 57.38 & 1430.1 \\
\hline & 13 & 2.9 & 190 & 3.50 & 0.38 & 21.15 & 1430.1 \\
\hline & 14 & 2.2 & 143 & 2.80 & 0.08 & 5.99 & 1430.1 \\
\hline & 15 & 1.8 & 108 & 2.30 & 0.73 & 50.48 & 1418.9 \\
\hline & 16 & 1.1 & 110 & 7.80 & 0.46 & 29.82 & 1409.2 \\
\hline & 17 & 2.9 & 177 & 4.60 & 0.42 & 26.19 & 1384.5 \\
\hline & 18 & 1.5 & 102 & 4.00 & 0.68 & 48.10 & 1458.5 \\
\hline & 19 & 2.3 & 162 & 6.20 & 0.23 & 12.06 & 1483.0 \\
\hline & 20 & 6.7 & 432 & 7.40 & 0.74 & 41.06 & 1455.1 \\
\hline & 21 & 3.0 & 179 & 5.40 & 0.72 & 49.02 & 1450.2 \\
\hline & 22 & 2.1 & 120 & 2.73 & 0.98 & 70.86 & 1463.0 \\
\hline & 23 & 3.7 & 226 & 5.79 & 0.62 & 44.45 & 1408.9 \\
\hline & 24 & 3.5 & 249 & 7.97 & 3.48 & 59.62 & 1525.3 \\
\hline & 25 & 1.2 & 100 & 5.52 & 1.18 & 36.29 & 1525.3 \\
\hline & 26 & 1.6 & 105 & 1.61 & 0.47 & 31.07 & 1666.9 \\
\hline & 27 & 2.6 & 168 & 3.24 & 0.19 & 9.50 & 1671.6 \\
\hline & 28 & 2.0 & 138 & 4.19 & 0.67 & 46.15 & 1626.2 \\
\hline & 29 & 1.7 & 110 & 3.64 & 0.19 & 15.26 & 1690.9 \\
\hline & 30 & 1.4 & 101 & 3.94 & 0.33 & 20.96 & 1690.9 \\
\hline \multirow{4}{*}{$\begin{array}{c}\text { spherical } \\
\text { particles } \\
\text { (Experiment) }\end{array}$} & 1 & & 122 & 4.36 & 0.08 & 5.46 & 1690.1 \\
\hline & 2 & & 95 & 2.58 & 0.33 & 21.33 & 1674.8 \\
\hline & 3 & & 108 & 3.94 & 0.45 & 30.75 & 1462.2 \\
\hline & 4 & & 95 & 2.54 & 0.75 & 51.50 & 1391.6 \\
\hline \multirow{4}{*}{$\begin{array}{c}\text { spherical } \\
\text { particles } \\
\text { (Simulation) }\end{array}$} & 1 & & 80 & 5.25 & 0.08 & 5.25 & 1600.0 \\
\hline & 2 & & 80 & 5.25 & 0.33 & 22.15 & 1600.0 \\
\hline & 3 & & 80 & 5.25 & 0.45 & 30.97 & 1600.0 \\
\hline & 4 & & 80 & 5.25 & 0.75 & 59.40 & 1600.0 \\
\hline
\end{tabular}

Annex 1: Short summary of particle properties. 


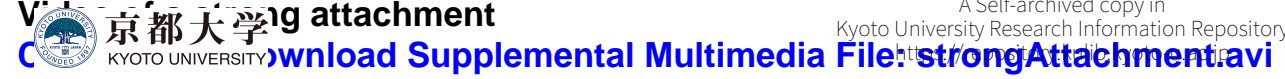

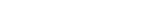

